\title{
Glis3 as a critical regulator of thyroid primordium specification
}

Giuditta Rurale ${ }^{1,2}$, PhD, Federica Marelli ${ }^{2}$, PhD, Paolo Duminuco ${ }^{1}$, BSc, Luca Persani ${ }^{1,2}$, MD

$\mathrm{PhD}$

${ }^{1}$ Department of Endocrine and Metabolic Diseases and Lab of Endocrine and Metabolic Research, Istituto Auxologico Italiano IRCCS, Milan 20149, Italy; ${ }^{2}$ Department of Clinical Sciences and Community Health, University of Milan, Milan 20122, Italy

Key words: Congenital Hypothyroidism; Thyroid Dysgenesis; Endoderm; Sonic Hedgehog; Thyroid Development; Zebrafish

Dr. Giuditta Rurale, PhD: giudi.rurale@gmail.com

Dr. Federica Marelli, PhD: federica.marelli@unimi.it

Dr. Paolo Duminuco, BSc: p.duminuco@auxologico.it

Prof. Luca Persani, MD PhD: luca.persani@unimi.it 


\section{Abstract}

Background: GLIS3 (GLI-Similar protein 3) is a transcription factor involved in several cellular processes. Homozygous mutations in the GLIS3 gene have been typically associated with Neonatal Diabetes and Congenital Hypothyroidism $(\mathrm{CH})$ in a syndrome called NDH. NDH patients present developmental abnormalities including endocrine pancreas defects and a spectrum of thyroid abnormalities, mainly including thyroid dysgenesis (TD). The mouse models revealed a key role of Glis3 in pancreatic islets but not in early thyroid development, as Glis3 was described to retain a role in regulating thyroid hormone synthesis downstream the TSH/TSHR signaling pathway and in postnatal follicle proliferation. Hence, in this study we have been taking advantage of the zebrafish model to gain insights on the Glis3 activity during thyroid organogenesis.

Methods: Transient glis3-knockdown zebrafish embryos (called glis3 morphants) were generated by the microinjection of specific glis3 morpholinos at 1-2 cells stage to analyze the thyroid phenotype in vivo. Several additional analyses (in situ hybridization, immunohistochemistry, pharmacological treatments) were performed for further molecular characterization.

Results: The analysis of thyroid embryonic development revealed that Glis3 is involved in early steps of thyroid specification. glis 3 morphants exhibited a reduced expression of the early transcription factors $n k x 2.4$ and $p a \times 2 a$ at the thyroid primordium level, that is not caused by changes in proliferation or apoptosis of the pharyngeal endoderm. As a result, the differentiated thyroid tissue in morphants appeared reduced in size with decreased expression of $t g$ and s/c5a5, a low number of T4-producting follicles, associated with an elevation of tshba (homologous of the human TSH ), thus resembling the clinical and biochemical manifestations of patients with TD. Interestingly, glis3 morphants have pancreatic $\beta$-cell defects, but not liver defects. In vitro and in vivo data also demonstrated that Glis3 is an effector of the Sonic Hedgehog (SHH) pathway. Molecular and pharmacological inhibition of $\mathrm{SHH}$ reproduced the thyroid defects observed in glis3 morphant. 
Conclusions: Our results demonstrate that glis3, within the SHH pathway, appears to determine the number of endodermal cells committed to a thyroid fate. This is the first evidence of the involvement of Glis3 in TD, thereby expanding the understanding of the genetic basis of thyroid development and $\mathrm{CH}$. 


\section{Introduction}

Several key steps of thyroid development have been understood owing to the seminal work of several groups that identified the set of thyroid transcription factors that define the thyroid precursor cells as well as differentiated thyrocytes, but the mechanism underlying the specification of the thyroid anlage from the foregut endoderm are currently unknown (1-3).

The expression of NKX2-1, PAX8, FOXE1 and HHEX is known to be required for adequate thyroid development and function in several models, including mice and zebrafish, and mutations affecting these genes have been associated with congenital hypothyroidism $(\mathrm{CH})$, one of the most common congenital endocrine diseases and the most prevalent preventable form of mental retardation (1-6).

Recent findings indicate that the transcription factor GLI-Similar protein 3 (GLIS3) is a candidate gene for $\mathrm{CH}$, although its role in thyroid pathophysiology remains largely unexplored. Homozygous and compound heterozygous mutations in GLIS3 have been associated with a rare syndrome, referred to as $\mathrm{NDH}$, characterized by neonatal diabetes (ND) and $\mathrm{CH}$, and several additional features, including renal, hepatic and skeletal abnormalities (6-8). All NDH cases have low T4 and high TSH levels; at the level of the thyroid, they are associated with a broad spectrum of clinical manifestations ranging from dysgenetic defects (from athyreosis to hypoplasia) to functional defects with an in situ thyroid gland. Moreover, we identified a number of heterozygous GLIS3 missense substitutions in a large cohort of patients with variable forms of $\mathrm{CH}$ (9). Interestingly, all of the GLIS3 variants are associated with other rare mutations in genes involved in $\mathrm{CH}$ pathology, thus supporting the concept of a frequent oligogenic origin of $\mathrm{CH}(5,6,9)$.

GLIS3 is a component of Krüppel-like zinc finger nuclear transcriptional regulators, which share a highly conservation of five $\mathrm{C}_{2}-\mathrm{H}_{2}$-type zinc finger motifs (10). GLIS3 plays critical roles in development and function of several organs, controlling gene transcription through the binding with GLIS3-binding sites (G3BS) present in the regulatory regions of target genes. In humans, GLIS3 is expressed in a tissue-specific manner, with abundant 
expression in thyroid, pancreas, and kidney. The GLIS3 gene generates several transcripts, some of which might have variable functional activities (10-12).

Up to now, the functional role of Glis3 has been extensively studied in mice. These monogenic diabetes models revealed that Glis3 is a fundamental player for endocrine pancreas differentiation from the endoderm, the definition of the $\beta$-cell mass, and that sustained postnatal expression is required for adequate insulin secretion and glucose metabolism $(10,13-16)$. In this context, several findings support the concept that Glis3 is one of the transcription factors belonging to the Sonic-Hedgehog $(\mathrm{SHH})$ signaling pathway. In particular, the binding with the Suppressor of Fused (SUFU), a negative regulator of SHH, accounts for the stability and transactivation activities of GLIS3 (13-20).

As far as the thyroid gland is concerned, evidence from Glis3 knockout (KO) mice indicated that Glis3 is essential for TH biosynthesis and postnatal thyroid gland proliferation, acting down-stream of the TSH/TSHR system $(10,21)$. Although the size of the thyroid follicles is smaller in Glis3-KO pups in the first week after birth, the thyroid morphology and serum $\mathrm{TH}$ levels are similar compared to wild-type mice, suggesting that the development of $\mathrm{CH}$ in this particular Glis3-KO mouse is related to functional rather than developmental defects. These findings are in contrast to those observed in the NDH patients, in which thyroid dysgenesis is a common feature $(7-9,22)$.

In this study, we took advantage of the zebrafish model to characterize the role of Glis3 during early thyroid development. Zebrafish has emerged as a suitable model system in thyroid research, thanks to the high conservation of the molecular mechanisms involved in thyroid organogenesis, TH transport and metabolism, as well as TH action in target tissues (23-27). We previously described that the glis3 transcript is detectable early during zebrafish embryonic development, and its signal depicts the foregut endoderm pouches, the tissue that will give rise to endocrine organs (e.g. thyroid and pancreas) (28). In the present work, we describe the effects of glis3 knock-down (KD) in zebrafish. The multiple genetic and pharmacological manipulations demonstrate that glis3, within the sonichedgehog pathway, is a critical factor for the correct specification of thyroid primordium, likely regulating the number of endodermal cells committed to thyroid fate. 


\section{Materials and Methods}

\section{Ethical statement}

Current Italian national rules: no approval needs to be given for research on zebrafish embryos. Fish were maintained and raised according to EU regulations on laboratory animals (Directive 2010/63/EU).

\section{Zebrafish line and maintenance}

Wild-type adults ( $A B$ strain) were obtained from the Wilson lab, University College of London, UK, and maintained in a flow-through system in charcoal-filtered tap water at a constant temperature $\left(28 \pm 1^{\circ} \mathrm{C}\right)$, with a photoperiod of $14: 10$ (light:dark). Zebrafish embryos, obtained from natural spawning, were raised and maintained according to established techniques $(29,30)$, and staged according to morphological criteria (31). Beginning from 24 hours post fertilization (hpf), the embryos were cultured in fish water containing $0.002 \%$ of 1 -phenyl-2-thiourea (PTU, SIGMA) to prevent pigmentation, and $0.01 \%$ methylene blue to prevent fungal growth. Such a low dose of PTU $(0.002 \%)$ does not affect TH synthesis and the thyroxine concentrations are similar in treated and untreated fish (32). The Wild Type (WT) AB strain zebrafish line was used.

\section{Probes preparation and whole mount in situ hybridization (WISH)}

For glis3 (ENSDART00000142833.2) antisense digoxigenin (DIG) probe synthesis, a $1024 \mathrm{bp}$ template was generated by RT-PCR on total RNA extracted from pools of embryos at 26-48 hpf. The primers used for probe amplification are the following: FW: 5' TGGGAAAGGCTGTAACCTGA 3' and REV: 5' GGACACCTCAAACTGAAGCG 3' $(28,33)$. The PCR products were cloned into the pCRII-TOPO vector (Invitrogen, Carlsbad, CA) and the cDNAcontaining plasmids were enzymatically linearized and in vitro transcribed to antisense digoxigenin (DIG) riboprobes using T7 or SP6 with RNA polymerase (Roche).

The riboprobes against $n k \times 2.4$, pax2a, tg, slc5a5 (33), tshba (34), shha (35), foxa2 (36), is/1 (37), foxa3 (38), ptf1a (39), ins2 (40) mRNAs were used to evaluate thyroid, endoderm, liver and pancreas development and function. WISH experiments were performed according to Thisse et al. (41). Depending on the experiment, the DIG-probes were 
detected using anti-DIG-alkaline phosphatase (AP) and NBT/BCIP or Fast Blue. For the analysis of thyroid volume quantification (NBT/BCIP), the embryos were flat mounted, and the number of positive cells was calculated by dividing the area of positive staining by the average area of a single cell using ImageJ software (http:// rsb.info.nih.gov/ij/). The double fluorescent WISH was performed as previously described (42). The DIG- and FLUO-labelled probes were either revealed by Fast Blue and Fluorescein using the Perkin Elmer TSA kit. The fluorescent signals were detected by confocal microscopy and the fixed embryos were embedded with $80 \%$ glycerol and mounted on glass slides. Stacks were recorded using a 20x objective plus $2 x$ zoom (Nikon $\mathrm{C} 2+$ confocal system; Nikon) and the images were processed using Adobe Photoshop CS2.

\section{Loss-of function analysis}

The embryos were injected with a specific Morpholino (MO), a specific antisense oligonucleotide to transiently knock down the gene function.

The specific glis3 morpholino (GLIS3MO_SPL: 5' TTCTTGTTTTTACCTTTCATACCGC 3') was designed by Gene Tools (LLC, Philomath, OR), in order to recognize the splice donor site of the exon 2-intron 2 boundary of the pre-mRNA. The negative controls were generated by the injection of standard control morpholino (std ctrl-MO: 5' CCTCTTACCTCAGTTACAATTTATA 3') that targets the human 8-globin gene. Morpholinos were dissolved in Danieau's buffer $\left(58 \mathrm{mM} \mathrm{NaCl} ; 0.7 \mathrm{mM} \mathrm{KCl} ; 0.4 \mathrm{mM} \mathrm{MgSO}{ }_{4} \mathrm{H}_{2} \mathrm{O} ; 0,6 \mathrm{mM}\right.$ $\mathrm{Ca}\left(\mathrm{NO}_{3}\right)_{2} ; 5 \mathrm{mM}$ Hepes $\mathrm{pH}$ 7.2). Embryos were microinjected at the 1-2 cells stage and Rodamine dextran (Molecular Probes) was usually co-injected as a tracer.

The GLIS3MO_SPL was validated by RT and qRT -PCR. Total RNA was extracted from injected and control embryos at 1 and 2 days post-fertilization (dpf) using TRIzol ${ }^{\mathrm{TM}}$ (Thermo Fisher Scientific). The reaction was carried out following the protocol of GoScript $^{\mathrm{TM}}$ Reverse Transcription System (Promega). The cDNAs were then subjected to PCR amplification using GoTaq polymerase (Promega) following the manufacturer's instructions. Quantitative real-time PCR (qRT-PCR) was performed by $A B I$ PRISMTM 7900HT Fast Real-Time PCR System using SYBRGreen MasterMix (Invitrogen). A second glis3 splicing morpholino (43) (GLIS3_MO2: 5'ACCTGCTGCAAGAGATCAGTTAAAA 3') has 
been used to confirm the results obtained with the GLIS3MO_SPL. The shha_MO (44) (5'CAGCACTCTCGTCAAAAGCCGCATT3') was used to investigate the relationship between glis3 and Shh.

\section{Rescue experiments}

Full-length zebrafish wild type glis3 cDNA was cloned into the $\operatorname{pcs}^{+}$vector (Invitrogen), using the following primers: glis3CDS_FW: 5' ATGGACATGAATGGGAAAG $3^{\prime}$ and glis3CDS_REV: 5' TCAGCCTTCAGTGAACACACA 3'.

The mutated form of glis3 mRNA was generated introducing a point mutation GGA>TGA at position $1223 \mathrm{bp}$ that causes the formation of a premature stop codon upstream of the zinc-finger functional domain. Both constructs (WT and MUT) were linearized and in vitro transcribed using the mMessage mMachine Sp6 kit (Thermo Fisher Scientific), following the manufacturer's instructions. The GFP mRNA was used as control for glis3 mRNAs microinjection, which has no targets in zebrafish.

\section{Immunofluorescence}

The qualitative analysis of TH production was performed by immunofluorescence according to standard procedures using a rabbit anti-T4 BSA serum $(1: 1000$; ICN Biochemicals) and the AlexaFluor 555 anti-rabbit IgG as secondary antibody (1:500, Life Technologies) $(23,36)$.

To test proliferation and apoptosis, after WISH with $n k x 2.4$-DIG riboprobes and Fast Blue staining, embryos were stripped and rinsed in PBS. Anti-phospho-histone H3 (PH3) and antiactive caspase-3 (AC-3) antibodies (1:250; Cell Signaling) were used as primary antibodies, followed by incubation with an antirabbit IgG secondary antibody/AlexaFluor 488 (1:500; Life Technologies) (36).

\section{Immunocytochemistry}

The entire CDS of the zebrafish sufu and glis3 were amplified and cloned into the pEGFPC3 and pcDNA4-Myc/His, respectively. HeLa cells were transfected with $1 \mu \mathrm{g}$ of

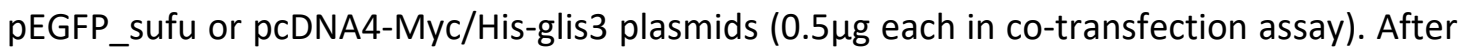


$24 \mathrm{~h}$, the transfected cells were fixed with 4\% paraformaldehyde (Sigma-Aldrich), permeabilized with $0.1 \%$ Triton X-100 (Sigma-Aldrich). For the detection of the glis3 construct, the cells were incubated o.n. with $\alpha$-c-Myc primary antibody (1:100; Life Technologies) and than detected with secondary antibody Alexa Fluor 555 goat anti-mouse IgG, (1:500 Life Technologies). The fixed cells were mounted with Slow-Fade Gold antifade reagent with DAPI (Life Technologies) and acquired using the Nikon Eclipse Ti confocal microscope equipped with a $60 \times$ oil immersion objective.

\section{Co-immunoprecipitation}

HeLa cells were transfected as previously described and after $24 \mathrm{~h}$, the total protein content was extracted using Cell Lysis Buffer $(20$ mM Tris- $\mathrm{HCl}$ pH 7.5, $150 \mathrm{mM} \mathrm{NaCl}, 1 \mathrm{mM}$ EDTA, 1 mM EGTA, 1\% Triton X-100, protease inhibitor cocktail (Roche)), and sonication. Protein lysates were quantified by BCA protein assay (Thermo Fisher Scientific) and $300 \mu \mathrm{g}$ of protein sample was incubated for 45 min RT with $450 \mu \mathrm{g}$ of Dynabeads ${ }^{\mathrm{TM}}$ Protein $\mathrm{G}$ (Invitrogen) conjugated to $1 \mu \mathrm{g}$ of anti-c-Myc Monoclonal Antibody (Invitrogen) or $1 \mu \mathrm{g}$ of normal mouse IgG (Santa Cruz Biotechnology). Magnetic beads were washed four times in PBS with $0.02 \%$ Tween-20, and resuspended in $20 \mu \mathrm{l} 2 \mathrm{x}$ sample loading buffer (50 mM Tris$\mathrm{HCL}$ pH 6.8, 2\% SDS, $0.1 \%$ bromophenol blue, 10\% glycerol) and 200 mM DTT (SigmaAldrich). Immunocomplexes and input fractions were examined by Western blot using 412\% NuPAGE Bis-Tris pre-cast polyacrylamide gels (Thermo Fisher Scientific). Immunoblots were performed with mouse c-Myc antibodies (1:500 Life Technologies) or mouse GFP antibody (1:1000, Origene) diluted in 5\% milk in TBS with 0.1\% Tween-20 (Sigma-Aldrich).

\section{Cyclopamine treatment}

For the inhibition of the Shh pathways, from $50 \%$ epiboly stage to 26 or $48 \mathrm{hpf}$, the embryos were treated with $10 \mu \mathrm{M}$ Cyclopamine (Sigma-Aldrich) harvested in fish water $(45,46) ; 1 \% \mathrm{DMSO}$ (dimethylsulfoxide) was used as a control vehicle.

\section{Statistical analysis}

All data are shown as means \pm standard deviation $(S D)$ or percentages. The $t$-student test was used for the analysis of differences between groups. The $p<0.05$ was considered 
statistically significant. All the analyses were conducted with the software package GraphPad Prism 4.0 (GraphPad, San Diego, CA).

\section{Results}

\section{glis3 knockdown affects the specification of thyroid primordium}

Knockdown experiments were performed by the microinjection of a specific glis 3 morpholino (glis3MO_SPL) into zebrafish embryos (called morphants). Following the setup of glis3 gene knockdown and embryonic phenotype analysis (see Supplementary, section 1), we performed whole mount in situ hybridization to test whether glis3 plays a role in zebrafish thyroid organogenesis.

At $1 \mathrm{dpf}$, the thyroid primordium was defective with a marked reduction of $n k x 2.4$ and pax $2 a$ expression in the glis3 morphants (Fig $1 \mathrm{~A}-\mathrm{C}^{\prime}$ and $\mathrm{E}-\mathrm{G}^{\prime}$ ). The $n k x 2.4$ was markedly reduced in the thyroid bud compared to controls in $65.6 \%$ of embryos injected with 0.3 pmol/e of glis3MO_SPL, and undetectable in almost all the embryos injected with 0.5 pmol/e of glis3MO_SPL (Fig 1D). Consistently, a reduced to absent pax2a expression was seen in $17.6 \%$ to $33.9 \%$, respectively, of the embryos injected with $0.3 \mathrm{pmol} / \mathrm{e} \mathrm{MO}$, and the expression of pax $2 a$ was reduced to absent in the $31.2 \%$ and $68.8 \%$ of morphants injected with $0.5 \mathrm{pmol} / \mathrm{e}$ (Fig 1E-H). When compared with the size in control embryos, the volume of the thyroid primordium was reduced by $50-95 \%$ in morphants (Fig 1 Graphs D' and $H^{\prime}$ ).

The mechanisms underlying the altered thyroid phenotype in the glis3-knockdown embryos was investigated by activated caspase-3 (AC-3) or phospho-histone H3 (PH3) immune-staining as markers of apoptosis or proliferation, respectively (Fig $1 \mathrm{l}-\mathrm{L}$ ). At $1 \mathrm{dpf}$, no significant differences in AC-3 (Fig I-J) or PH3 (Fig 1K-L) staining were seen by confocal microscopy in the area of $n k x 2.4$ expression in all morphants. These findings indicate that the reduced expression of the early thyroid markers is not associated with a decreased proliferation nor an increased apoptosis of the endoderm precursors committed to thyroid fate. In aggregate, these findings point to the potential role of glis 3 in regulating the amount of endoderm cells that will be addressed to thyroid precursors. 
glis3 morphants have thyroid dysgenesis and primary hypothyroidism

At $2 \mathrm{dpf}$, the precursors of thyroid primordium differentiate into thyroid follicles, expressing the functional markers $t g$ and $s / c 5 a 5$, and proliferation along the ventral aorta by $3 \mathrm{dpf}(26)$. In agreement with the findings reported above, the injection of $0.3 \mathrm{pmol} / \mathrm{e}$ or $0.5 \mathrm{pmol} / \mathrm{e} \mathrm{MO}$ caused a marked and dose-dependent reduction of all the examined markers. The $t g$ transcript was reduced in $51.7 \%$ and $74.4 \%$ of morphants, respectively (Fig $2 \mathrm{~A}-\mathrm{C})$. Similarly, the s/c5a5 transcript was reduced, frequently below the limit of detection, in 74 or $88 \%$ of morphants (Fig $2 \mathrm{D}-\mathrm{G}$ ). At $3 \mathrm{dpf}$, the thyroid gland elongates along the midline forming the classical V-shape (Fig $2 \mathrm{H}$ ): the staining of $t g$ continued to be reduced in 32 or $50 \%$ of the morphants (Fig $2 \mathrm{I}$ and K). Interestingly, a significant portion of morphants exhibited a normal expression of $t g$, but their thyroid follicles were disorganized and ectopically displaced along the ventral aorta (Fig $2 \mathrm{~J}$ and $\mathrm{K}$ ).

The quantification of tg+ cell number confirmed the distribution of the thyroid phenotype of glis3 morphants at 2 and $3 \mathrm{dpf}$ (Fig $2 \mathrm{~L}$ and $\mathrm{M}$ ).

A double fluorescent WISH of early and late thyroid markers was also performed to understand the variable degree of impairment detected in the in glis3 morphants up to 60 hpf (see Supplementary, section 2). Most of the embryos presented a significant reduction of both early transcripts $(n k \times 2.4$ and pax2a), but pax2a was generally diminished to a larger extent. However, when the expression was completely absent, no thyroid bud was present and all markers were affected.

Immune-staining with an anti-T4 antibody was performed to assess the status of thyroid function at $5 \mathrm{dpf}$ by comparing the control and morphants injected with $0.5 \mathrm{pmol} / \mathrm{e}$. By counting individual T4-positive follicles, we observed that the glis3 morphants exhibited a significantly $(p<0.01)$ reduced number of follicles ( $n: 3 \pm 1$ vs $5 \pm 1$ ) (Fig $2 N-P)$. Consistently, $85 \%$ of the glis3 morphants presented a significant increase $(p<0.001)$ in the number of the tshba (homologous of human TSHB) positive pituitary cells at $5 \mathrm{dpf}(\mathrm{n}: 13 \pm 2 \mathrm{vs} 4 \pm 1$ ) (Fig 2Q-S).

The low T4 production and the high tshba expression observed in glis3-knockdown larvae well recapitulate the clinical findings described in patients with thyroid dysgenesis. The 
thyroid phenotype in glis3-deficient embryos was faithfully confirmed by using a second glis3 splicing morpholino that had been previously validated (43) (see Supplementary, section 3).

\section{The glis3 gene dosage regulates the number of the differentiated thyroid follicles}

To confirm the specific thyroid phenotype resulting from glis3 morpholino activity, we performed mRNA rescue experiments co-injecting the glis3MO_SPL with the wild-type or mutated zebrafish glis3 transcripts. The mutant glis3 mRNA was generated introducing a point mutation GGA>TGA at position 1223, that causes the formation of a premature stop codon upstream of the ZF domain, mimicking natural human GLIS3 mutations (7).

The co-injection with the glis3MO_SPL and the glis3_WT mRNA was able to rescue the loss of expression of $n k \times 2.4$ and $t g$ (Fig 3D and $D^{\prime}$ ) induced in the vast majority of the embryos injected with glis3MO_SPL alone (Fig $3 \mathrm{C}$ and $\mathrm{C}^{\prime}$ ). Interestingly, the co-injection of the glis3MO_SPL and the glis3_MUT mRNA (Fig 3E and E') failed to rescue the thyroid defects of glis3 morphants and even worsened the phenotype. The thyroid was not affected after the injection of the same doses (100 pg/e) of WT or MUT glis3 mRNAs (Fig 3B and B').

To further define whether glis3 gene dosage contributes to the onset of thyroid defects, we performed over-expression experiments microinjecting growing doses of glis3_WT mRNA. When compared with controls at $2 \mathrm{dpf}$ (Fig 3F), the injection of $250 \mathrm{pg} / \mathrm{e}$ of the glis3_WT mRNA was associated with an upregulation of $t g$ expression in $75 \%$ of embryos, and an enlargement of the thyroid anlage ( $+130 \%$ than controls) (Fig $3 \mathrm{H}$ and $\mathrm{I}$ ). Confirming the previous results, the thyroid was smaller in $87 \%$ of glis 3 morphants compared to controls (Fig 3G and I), and the number of T4-producing follicles was reduced by $50 \%$ (Fig $3 \mathrm{~F}^{\prime}$ and $\mathrm{G}^{\prime}$ ). In contrast, the overexpression of glis3 led to a significant increase of the T4 production (Fig $3 \mathrm{H}^{\prime}$ and $\mathrm{I}^{\prime}$ ).

To test whether the increased size of the thyroid gland in the glis3-overexpressing embryos was caused by an increased proliferation in the foregut endoderm or an enlarged number of endodermal cells committed to the thyroid fate, we performed proliferation assays at different time points. Since no differences in phospho-histone $\mathrm{H} 3$ levels were detected in the pharyngeal floor in early and late developmental stages after the injection 
of $250 \mathrm{pg} / \mathrm{e}$ glis3_WT mRNA (Fig 3J-O), these data also support the concept that the amount of glis3 activity is critical for the determination of thyroid primordium size.

\section{glis3 regulates the development of the endoderm-derived endocrine organs}

In order to exclude a global impairment of the pharyngeal endoderm in the glis3 morphants, we analyzed the expression of foxa2, an early endoderm-specific transcription factor. When thyroid bud becomes evident (22hpf), the endoderm pouches appeared unaffected in glis3 morphants (Fig 4A and B).

Furthermore, we investigated whether other endoderm-derived organs, such as liver and pancreas, show developmental defects in glis3 morphants. Liver development was unaltered in $100 \%$ of the glis 3 morphants, as shown in the WISH analysis of the expression of the liver-specific genes is/1 and foxa3 (Fig 4C-F). In contrast, the glis3 morphants showed endocrine pancreas defects (Fig 4G-I). In 52\% of morphants we observed an impaired ins 2 expression at $2 \mathrm{dpf}$ (Fig $4 \mathrm{H}$ ), and in the remaining $48 \%$ the ins 2 expression was disorganized, i.e. the insulin2-positive cells failed to converge on the midline to form a single islet (Fig 4I). The WISH of the acinal specific marker ptf1a also confirmed that the pancreatic defects of morphants were restricted to the endocrine compartment (see Supplementary, section 4).

These results suggest that glis 3 action is required for the correct development of the thyroid and the endocrine pancreas, but its down-regulation does affect the formation of pharyngeal endoderm pouches and liver development.

\section{glis3 acts as an effector of the sonic-hedgehog pathway}

Preliminary in vitro assays confirmed that sufu and glis3 interact even in zebrafish, since they co-localized and co-immunoprecipitated in a protein complex (see Supplementary, section 5).

To study the relationship between glis3 and Shh, the expression of shha was analyzed in glis3 morphants by WISH. When compared with controls at $2 \mathrm{dpf}$ (Fig $5 \mathrm{~A}$ and B), the 100\% of glis3 morphants injected with $0.5 \mathrm{pmol} / \mathrm{e}$, presented a significant reduction of shha expression in the pharyngeal endoderm (Fig 5C and D). 
The expression of glis3 was previously shown to be expressed early in the pharyngeal endoderm and absent in the differentiated thyroid (28). Here, we show that the downregulation of the shh pathway by injection of shha_MO (1.25 pmol/e) (44) or treatment with cyclopamine $(10 \mu \mathrm{M})(45,46)$ is associated with the lack of glis3 expression in both brain and pharyngeal endoderm (Fig 5E-L).

To test whether Shh signalling plays a role in thyroid development, the expression of endodermal and thyroid markers was analyzed by WISH in the cyclopamine treated embryos. In agreement with the key role of the shh pathway during endoderm formation and development, foxa 2 was compromised in the cyclopamine-treated embryos (Fig 6A and B). As a consequence of the pharmacological inhibition of the shh pathway, thyroid development was severely compromised. In fact, at $1 \mathrm{dpf}, n k \times 2.4$ and pax2a signals were reduced/absent at the level of thyroid primordium in the $100 \%$ of the cyclopamine-treated embryos (Fig 6C-F), and at 2 and $3 \mathrm{dpf}$, $t$ g expression was reduced in $68 \%$ or absent in $32 \%$ of treated-embryos (Fig 6G-L).

The overexpression of glis3 after the injection of WT glis3 mRNA (250 pg/e) did not alter shha transcript levels at $2 \mathrm{dpf}$ (Fig. 6M and $\mathrm{N}$ ), but enhanced the number of endodermal precursor cells toward the thyroid fate, thus resulting in an excess of $t g$-expressing cells (Fig 3). Surprisingly, cyclopamine treatment is able to completely counteract the effects of glis3-overexpression, and thus negatively affects thyroid development (Fig 60-Q). Accordingly, glis3 requires shh signaling for its activity and is likely to be a main downstream effector of this pathway in thyroid specification.

\section{Discussion}

Here, we demonstrate that the down-regulation of glis3 in zebrafish embryos leads to developmental defects in the thyroid, replicating the thyroid dysgenesis reported in several patients with GLIS3 mutations. The step-by-step analysis of thyroid organogenesis reveals that glis3 knockdown interferes with the specification of thyroid precursors from the pharyngeal endoderm, as shown by the absent or reduced expression of the early transcription factors $n k \times 2.4$ and pax $2 a$ at $1 \mathrm{dpf}$. Interestingly, at early stages of development the glis3 knockdown appears to affect pax2a to an extent larger than $n k x 2.4$. 
However, at later stages (2-3 dpf), glis3 morphants show a reduced or absent expression of $\operatorname{tg}$ and slc5a5, two of the key genes involved in thyroid hormone synthesis. As a consequence of these developmental defects, the glis3 morphant larvae present thyroid hypoplasia, a reduced number of T4-producing follicles, and high levels of thsba at $5 \mathrm{dpf}$, thus recapitulating the primary hypothyroidism observed in NDH patients.

Importantly, because no variations in proliferation or apoptosis were detected under glis 3 knockdown in the region including the thyroid bud, our findings support the concept that glis3 action is fundamental for the quantitative regulation of the amount of endodermal precursors committed to a thyroid fate. Consistently, the recovery of the thyroid phenotype in the rescue experiment, as well as the opposite variation in the number of thyroid cells and T4-production obtained through overexpression of the glis3 mRNA, confirms that the gene dosage of glis3 is critical for correct development and size definition of the zebrafish thyroid. These effects appear independent of the tsh/tshr signal since these transcripts can be detected in zebrafish from $40-42 \mathrm{hpf}(24,26)$, and tshba was found to be high in glis3 morphants. Previous work on zebrafish shh mutants reported an impaired pituitary gland and absence of tshba positive cells (47-49). This discrepant effect on pituitary markers may perhaps be explained by the involvement of different downstream effectors of the Shh pathway in pituitary and thyroid development: Glis3 may have a more relevant role in thyroid (and endocrine pancreas) development, whereas other Gli-like factors may exert a main developmental role in the pituitary.

Interestingly, the pharyngeal endoderm appeared unaffected in glis3 morphants (see Fig 4), thus excluding that the observed thyroid defects are a consequence of aberrant endoderm formation. Accordingly, we investigated whether other organs that arise from the endoderm (e.g. liver, exocrine and endocrine pancreas) display some developmental defects. Only the endocrine pancreas development appears to be affected by glis 3 knockdown, as glis3 morphants display a reduced differentiation of the ins2-positive pancreatic $\beta$-cells that frequently fail to migrate and localize in a disorganized shape. This phenotype displays similarities with the previously reported alterations of endocrine pancreas differentiation in Glis3-deficient conditions $(10,18,21,50)$. 
16

The decrease of the functional thyroid markers during glis3 knockdown at 2-3 dpf could be the consequence of direct or indirect effects. Indeed, PAX8 and NKX2.1 were shown to regulate the transcription of thyroidal genes involved in thyroid hormone synthesis through the direct binding of their promoter regions $(1,2,21)$, however CHIP-sequencing of mice thyroid tissues identified $P a x 8, P d s$, and $S / c 5 a 5$ as direct transcriptional targets of Glis3, thus pointing to a direct regulatory role of Glis3 on thyroid function. In apparent contrast with our findings, transcriptome analysis showed that expression of Pax8 and Nkx2.1 was unchanged in Glis3KO mice one month after birth (21). This discrepancy might be explained by the existence of compensatory mechanisms (e.g. other Gli-like activities) that are able to rescue, at least partially, Glis3 deficiency in the murine thyroid. In partial agreement with our findings, Glis3KO pups tend to have smaller thyroid glands than wildtype littermates (21). In aggregate, these findings support the concept of a dual role for glis3 on thyroid gland activity by promoting its commitment from the pharyngeal endoderm and, later on, the function/proliferation of thyroid cells. Indeed, Glis3 was previously reported to have a dual role also in pancreatic function: a) by regulating the number of endocrine progenitors that will differentiate into $\beta$-cells during embryonic growth, and b) by postnatally mediating insulin secretion and $\beta$-cell proliferation (13-19, 51).

The post-translational stability and transcriptional activity of GLIS3 were reported to be regulated by the binding with the Suppressor of Fused (SUFU), a negative regulator of the Sonic-hedgehog (SHH) signaling $(20,52,53)$. In absence of SHH, SUFU would anchor GLIS3 in the cytoplasm preventing its interaction with the ubiquitin ligase CUL3 and ITCH, thus protecting GLIS3 from proteasome degradation $(20,54)$. On the other hand, the GLIS3SUFU complex was also reported to translocate into the nucleus, where it recruits and interacts with co-repressors, suppressing the transcription of some target genes (e.g. Ins2) $(13,17,20,55)$. Through in vitro experiments, we then found that the zebrafish proteins glis3 and sufu co-localized in the nuclei of transfected cells and precipitate together in a co-IP assay, thus confirming that the interplay between glis3 and shh signaling occurs also in zebrafish. Subsequently, we observed that the shha signal is significantly reduced in the pharyngeal endoderm of glis3 morphants, and coherently, the expression of glis 3 is 
abolished in shh-deficient embryos (shha morphants and cyclopamine-treated embryos). Altogether these data point to a relevant developmental role of the shha-glis3 pathway in the pharyngeal endoderm and suggest that shh activity would induce expression of glis3 in the zebrafish endoderm.

Moreover, embryos treated with the shha antagonist developed a thyroid phenotype characterized by reduced/absent expression of early and late markers, a phenotype similar to that described in glis3 morphants. Interestingly, cyclopamine treatment also prevents the $t g$ up-regulation induced by glis3-overexpression. Therefore, glis3 appears to require an active Shh-signal for its function, likely acting as a downstream effector of the Shhpathway during zebrafish thyroid development.

In conclusion, we propose that glis3 acts as an effector of the Shh-pathway with a critical role during embryonic thyroid development. In particular, our data provide significant insights into the signaling pathways involved in the induction of thyroid precursors from the undifferentiated endoderm. However, additional experiments are needed to characterize the molecular events linking the shh-glis3 pathway and the expression and action of early thyroid transcription factors. Our findings may provide an explanation for the frequent developmental thyroid defects in patients with GLIS3 mutations. Finally, zebrafish may represent an alternative in vivo model suitable for high-throughput screening of drugs able to counteract GLIS3 deficiency (50).

\section{Acknowledgments}

The Authors wish to thank Dr Annamaria Maraschi (Milan) for the supply of pEGFP-C3 and pcDNA4-Myc/His constructs and for help with the setting of the experimental protocols for in vitro studies.

Correspondence: Prof Luca Persani, MD PhD; email: luca.persani@unimi.it

\section{Author's Contributions and Disclosure Statement}

GR designed and performed the in vivo experiments, and wrote the draft of the manuscript, and. FM supervised and contributed the zebrafish studies and the manuscript preparation. PD performed the in vitro experiments. LP conceived the study, obtained 
research funds, revised and finalized the manuscript. All authors approved the final version of the manuscript.

Funding: This work has been partially supported by the Ricerca Corrente funds of Istituto Auxologico Italiano, Milan, Italy (code: 05C102_2011) and by the PhD and Post-Doc programs of the University of Milan, Italy. 


\section{References}

1. De Felice M, Di Lauro R 2004 Thyroid development and its disorders: genetics and molecular mechanisms. Endocr Rev 25:722-746.

2. De Felice M, Di Lauro R 2007 Murine models for the study of thyroid gland development. Endocr Dev 10:1-14.

3. Fagman $\mathrm{H}$, Amendola $\mathrm{E}$, Parrillo L, Zoppoli $\mathrm{P}$, Marotta $\mathrm{P}$, Scarfo $\mathrm{M}$, De Luca $\mathrm{P}$, de Carvalho DP, Ceccarelli M, De Felice M, Di Lauro R 2011 Gene expression profiling at early organogenesis reveals both common and diverse mechanisms in foregut patterning. Dev Biol 359:163-175.

4. Szinnai G, Lacroix L, Carre A, Guimiot F, Talbot M, Martinovic J, Delezoide AL, Vekemans M, Michiels S, Caillou B, Schlumberger M, Bidart JM, Polak M 2007 Sodium/iodide symporter (NIS) gene expression is the limiting step for the onset of thyroid function in the human fetus. J Clin Endocrinol Metab 92:70-76.

5. Persani L, Rurale G, de Filippis T, Galazzi E, Muzza M, Fugazzola L 2018 Genetics and management of congenital hypothyroidism. Best practice \& research Clin Endocrinol Metab 32:387-396.

6. Peters C, van Trotsenburg ASP, Schoenmakers N 2018 Diagnosis of endocrine disease: Congenital hypothyroidism: update and perspectives. Eur J Endocrinol 179:R297R317.

7. Dimitri P 2017 The role of GLIS3 in thyroid disease as part of a multisystem disorder. Best Pract Res Clin Endocrinol Metab 31:175-182.

8. Senee V, Chelala C, Duchatelet S, Feng D, Blanc H, Cossec JC, Charon C, Nicolino M, Boileau P, Cavener DR, Bougneres P, Taha D, Julier C 2006 Mutations in GLIS3 are responsible for a rare syndrome with neonatal diabetes mellitus and congenital hypothyroidism. Nat Genet 38:682-687.

9. de Filippis T, Gelmini G, Paraboschi E, Vigone MC, Di Frenna M, Marelli F, Bonomi M, Cassio A, Larizza D, Moro M, Radetti G, Salerno M, Ardissino D, Weber G, Gentilini D, Guizzardi F, Duga S, Persani L 2017 A frequent oligogenic involvement in congenital hypothyroidism. Hum Mol Genet 26:2507-2514. 
10. Jetten AM 2018 GLIS1-3 transcription factors: critical roles in the regulation of multiple physiological processes and diseases. Cell Mol Life Sci 75:3473-3494.

11. Kim YS, Nakanishi G, Lewandoski M, Jetten AM 2003 GLIS3, a novel member of the GLIS subfamily of Kruppel-like zinc finger proteins with repressor and activation functions. Nucleic Acids Res 31:5513-5525.

12. Lichti-Kaiser K, ZeRuth G, Jetten AM 2014 Transcription Factor Gli-Similar 3 (Glis3): Implications for the Development of Congenital Hypothyroidism. J Endocrinol Diabetes Obes 2:1024.

13. Kang HS, Kim YS, ZeRuth G, Beak JY, Gerrish K, Kilic G, Sosa-Pineda B, Jensen J, Pierreux CE, Lemaigre FP, Foley J, Jetten AM 2009 Transcription factor Glis3, a novel critical player in the regulation of pancreatic beta-cell development and insulin gene expression. Mol Cell Biol 29:6366-6379.

14. Watanabe N, Hiramatsu K, Miyamoto R, Yasuda K, Suzuki N, Oshima N, Kiyonari H, Shiba D, Nishio S, Mochizuki T, Yokoyama T, Maruyama S, Matsuo S, Wakamatsu Y, Hashimoto H 2009 A murine model of neonatal diabetes mellitus in Glis3-deficient mice. FEBS Lett 583:2108-2113.

15. Yang Y, Chang BH, Chan L 2013 Sustained expression of the transcription factor GLIS3 is required for normal beta cell function in adults. EMBO Mol Med 5:92-104.

16. Kang HS, Takeda Y, Jeon K, Jetten AM 2016 The Spatiotemporal Pattern of Glis3 Expression Indicates a Regulatory Function in Bipotent and Endocrine Progenitors during Early Pancreatic Development and in Beta, PP and Ductal Cells. PloS One 11:e0157138.

17. Hang Y, Stein R 2011 MafA and MafB activity in pancreatic beta cells. Trends Endocrinol Metab 22:364-373.

18. Yang Y, Chang BH, Samson SL, Li MV, Chan L 2009 The Kruppel-like zinc finger protein Glis3 directly and indirectly activates insulin gene transcription. Nucleic Acids Res 37:2529-2538.

19. ZeRuth GT, Takeda Y, Jetten AM 2013 The Kruppel-like protein Gli-similar 3 (Glis3) functions as a key regulator of insulin transcription. Mol Endocrinol 27:1692-1705.

20. ZeRuth GT, Yang XP, Jetten AM 2011 Modulation of the transactivation function and stability of Kruppel-like zinc finger protein Gli-similar 3 (Glis3) by Suppressor of Fused. J Biol Chem 286:22077-22089. 
21. Kang HS, Kumar D, Liao G, Lichti-Kaiser $K$, Gerrish $K$, Liao XH, Refetoff $S$, Jothi R, Jetten AM 2017 GLIS3 is indispensable for TSH/TSHR-dependent thyroid hormone biosynthesis and follicular cell proliferation. J Clin Invest 127:4326-4337.

22. Dimitri P, Habeb AM, Gurbuz F, Millward A, Wallis S, Moussa K, Akcay T, Taha D, Hogue J, Slavotinek A, Wales JK, Shetty A, Hawkes D, Hattersley AT, Ellard S, De Franco E 2015 Expanding the Clinical Spectrum Associated With GLIS3 Mutations. J Clin Endocrinol Metab 100:E1362-1369.

23. Wendl T, Lun K, Mione M, Favor J, Brand M, Wilson SW, Rohr KB 2002 Pax2.1 is required for the development of thyroid follicles in zebrafish. Development 129:37513760 .

24. Porazzi P, Calebiro D, Benato F, Tiso N, Persani L 2009 Thyroid gland development and function in the zebrafish model. Mol Cell Endocrinol 312:14-23.

25. Opitz R, Maquet E, Huisken J, Antonica F, Trubiroha A, Pottier G, Janssens V, Costagliola S 2012 Transgenic zebrafish illuminate the dynamics of thyroid morphogenesis and its relationship to cardiovascular development. Dev Biol 372:203-216.

26. Opitz R, Antonica F, Costagliola S 2013 New model systems to illuminate thyroid organogenesis. Part I: an update on the zebrafish toolbox. Eur Thyroid J 2:229-242.

27. Marelli F, Persani L 2017 How zebrafish research has helped in understanding thyroid diseases. F1000Res 6:2137-2146.

28. Rurale G, Persani L, Marelli F 2018 GLIS3 and Thyroid: A Pleiotropic Candidate Gene for Congenital Hypothyroidism. Front Endocrinol (Lausanne) 9:730-737.

29. Fishman MC, Stainier DY, Breitbart RE, Westerfield M 1997 Zebrafish: genetic and embryological methods in a transparent vertebrate embryo. Methods Cell Biol 52:67-82.

30. Tsang B, Zahid H, Ansari R, Lee RC, Partap A, Gerlai R 2017 Breeding Zebrafish: A Review of Different Methods and a Discussion on Standardization. Zebrafish 14:561-573.

31. Kimmel CB, Ballard WW, Kimmel SR, Ullmann B, Schilling TF 1995 Stages of embryonic development of the zebrafish. Dev Dyn 203:253-310.

32. Marelli F, Carra S, Agostini M, Cotelli F, Peeters R, Chatterjee K, Persani L 2016 Patterns of thyroid hormone receptor expression in zebrafish and generation of a novel model of resistance to thyroid hormone action. Mol Cell Endocrinol 424:102-117. 
33. Alt B, Reibe S, Feitosa NM, Elsalini OA, WendI T, Rohr KB 2006 Analysis of origin and growth of the thyroid gland in zebrafish. Dev Dyn 235:1872-1883.

34. Opitz R, Maquet E, Zoenen M, Dadhich R, Costagliola S 2011 TSH receptor function is required for normal thyroid differentiation in zebrafish. Mol Endocrinol 25:1579-1599.

35. dilorio P, Alexa K, Choe SK, Etheridge L, Sagerstrom CG 2007 TALE-family homeodomain proteins regulate endodermal sonic hedgehog expression and pattern the anterior endoderm. Dev Biol 304:221-231.

36. Porazzi $P$, Marelli F, Benato F, de Filippis $T$, Calebiro D, Argenton F, Tiso N, Persani $L$ 2012 Disruptions of global and JAGGED1-mediated notch signaling affect thyroid morphogenesis in the zebrafish. Endocrinology 153:5645-5658.

37. Korzh V, Edlund T, Thor S 1993 Zebrafish primary neurons initiate expression of the LIM homeodomain protein IsI-1 at the end of gastrulation. Development 118:417-425.

38. Odenthal J, Nusslein-Volhard C 1998 fork head domain genes in zebrafish. Dev Genes Evol 208:245-258.

39. Zecchin E, Mavropoulos A, Devos N, Filippi A, Tiso N, Meyer D, Peers B, Bortolussi M, Argenton F 2004 Evolutionary conserved role of ptf1a in the specification of exocrine pancreatic fates. Dev Biol 268:174-184.

40. Biemar F, Argenton F, Schmidtke R, Epperlein S, Peers B, Driever W 2001 Pancreas development in zebrafish: early dispersed appearance of endocrine hormone expressing cells and their convergence to form the definitive islet. Dev Biol 230:189-203.

41. Thisse C, Thisse B 2008 High-resolution in situ hybridization to whole-mount zebrafish embryos. Nat Protocols 3:59-69.

42. Lauter G, Soll I, Hauptmann G 2011 Two-color fluorescent in situ hybridization in the embryonic zebrafish brain using differential detection systems. BMC Dev Biol 11:43-54.

43. O'Hare EA, Yerges-Armstrong LM, Perry JA, Shuldiner AR, Zaghloul NA 2016 Assignment of Functional Relevance to Genes at Type 2 Diabetes-Associated Loci Through Investigation of beta-Cell Mass Deficits. Mol Endocrinol 30:429-445.

44. Zhang C, Ojiaku P, Cole GJ 2013 Forebrain and hindbrain development in zebrafish is sensitive to ethanol exposure involving agrin, Fgf, and sonic hedgehog function. Birth defects research Part A, Clinical and molecular teratology 97:8-27. 
45. Chen JK, Taipale J, Cooper MK, Beachy PA 2002 Inhibition of Hedgehog signaling by direct binding of cyclopamine to Smoothened. Genes Dev 16:2743-2748.

46. Incardona JP, Gaffield W, Kapur RP, Roelink H 1998 The teratogenic Veratrum alkaloid cyclopamine inhibits sonic hedgehog signal transduction. Development 125:35533562.

47. Devine CA, Sbrogna JL, Guner B, Osgood M, Shen MC, Karlstrom RO 2009 A dynamic Gli code interprets Hh signals to regulate induction, patterning, and endocrine cell specification in the zebrafish pituitary. Dev Biol 326:143-154.

48. Herzog W, Zeng X, Lele Z, Sonntag C, Ting JW, Chang CY, Hammerschmidt M 2003 Adenohypophysis formation in the zebrafish and its dependence on sonic hedgehog. Dev Biol 254:36-49.

49. Sbrogna JL, Barresi MJ, Karlstrom RO 2003 Multiple roles for Hedgehog signaling in zebrafish pituitary development. Dev Biol 254:19-35.

50. Amin S, Cook B, Zhou T, Ghazizadeh Z, Lis R, Zhang T, Khalaj M, Crespo M, Perera M, Xiang JZ, Zhu Z, Tomishima M, Liu C, Naji A, Evans T, Huangfu D, Chen S 2018 Discovery of a drug candidate for GLIS3-associated diabetes. Nat Commun 9:2681-2693.

51. Yang Y, Bush SP, Wen X, Cao W, Chan L 2017 Differential Gene Dosage Effects of Diabetes-Associated Gene GLIS3 in Pancreatic beta Cell Differentiation and Function. Endocrinology 158:9-20.

52. Li B, Rauhauser AA, Dai J, Sakthivel R, Igarashi P, Jetten AM, Attanasio M 2011 Increased hedgehog signaling in postnatal kidney results in aberrant activation of nephron developmental programs. Hum Mol Genet 20:4155-4166.

53. Rual JF, Venkatesan K, Hao T, Hirozane-Kishikawa T, Dricot A, Li N, Berriz GF, Gibbons FD, Dreze M, Ayivi-Guedehoussou N, Klitgord N, Simon C, Boxem M, Milstein S, Rosenberg J, Goldberg DS, Zhang LV, Wong SL, Franklin G, Li S, Albala JS, Lim J, Fraughton C, Llamosas E, Cevik S, Bex C, Lamesch P, Sikorski RS, Vandenhaute J, Zoghbi HY, Smolyar A, Bosak S, Sequerra R, Doucette-Stamm L, Cusick ME, Hill DE, Roth FP, Vidal M 2005 Towards a proteome-scale map of the human protein-protein interaction network. Nature 437:1173-1178. 
54. ZeRuth GT, Williams JG, Cole YC, Jetten AM 2015 HECT E3 Ubiquitin Ligase Itch Functions as a Novel Negative Regulator of Gli-Similar 3 (Glis3) Transcriptional Activity. Plos One 10:e0131303.

55. Chou CK, Tang CJ, Chou HL, Liu CY, Ng MC, Chang YT, Yuan SF, Tsai EM, Chiu CC 2017 The Potential Role of Kruppel-Like Zinc-Finger Protein Glis3 in Genetic Diseases and Cancers. Arch Immunol Ther Exp (Warsz) 65:381-389. 


\section{Figure Legends}

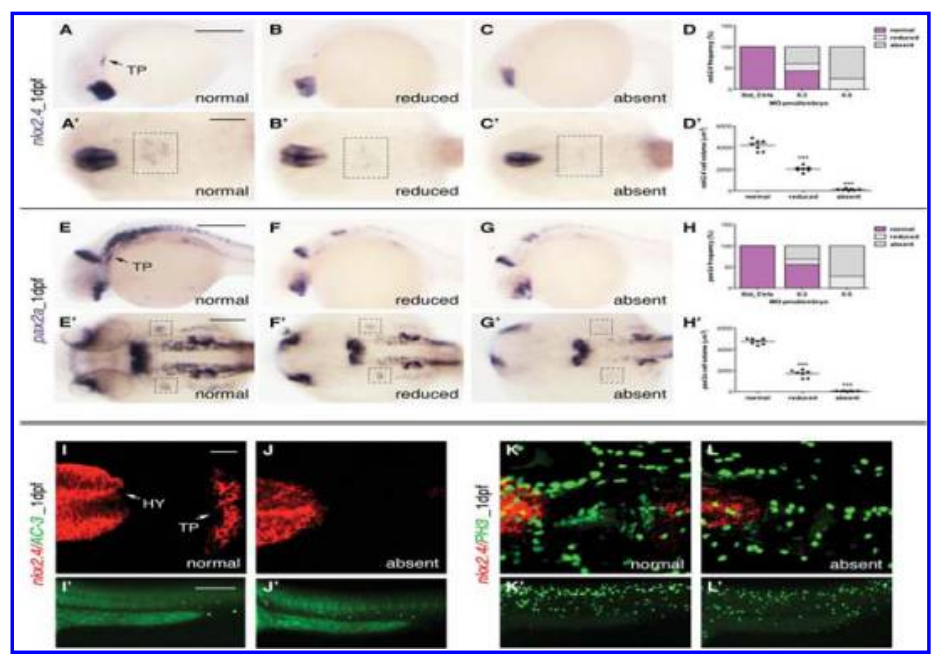

Figure 1. Analysis of the developing thyroid primordium (TP). (A-C and $A^{\prime}-C^{\prime}$ ) WISH of nkx2.4 expression in embryos injected with Std-CtrlMO and glis3MO_SPL at 0.3 and $0.5 \mathrm{pmol} / \mathrm{e}$. Images are representative of injected embryos that expressed normal, reduced or absent $n k \times 2.4$ signal acquired in lateral and dorsal view. (E-G and $\mathbf{E}^{\prime}-\mathbf{G}^{\prime}$ ) $p a \times 2 a$ expression in the injected embryos at $1 \mathrm{dpf}$. The embryos were subdivided belonging the three phenotypic classes described above. Scale bar: $250 \mu \mathrm{m}$. (Graphs D, H) Bars indicate the percentages of the injected embryos belonging the different phenotypic classes. (Graphs D', H') Quantification of $n k x 2.4$ and pax2a at $1 \mathrm{dpf}$ in the control and morphant embryos belonging the thyroid phenotypic classes. Data were obtained from 10 embryos for each class. Statistical significance was calculated using Student's t-test (***P<0.001). (IL) WISH of nkx2.4, at 1dpf, using FastBlue staining for the acquisition of the fluorescence signal, followed by activated caspase-3 (AC-3) (I-J) and phospho-histone H3 (PH3) (K-L) immunostaining. Std-ctrl embryos reveal the correct development of the thyroid primordium. In glis3-morphants the thyroid primordium is absent. No differences in AC-3 or in $\mathrm{PH} 3$ immunostaining were observed in morphants, compared to controls. Embryos are all flat mounted, in ventral view, with the anterior to the left. Scale bar: $100 \mu \mathrm{m}$. (I'-J' and $\left.\mathbf{K}^{\prime}-\mathrm{L}^{\prime}\right)$ AC-3 and $\mathrm{PH} 3$ staining of the trunktail regions were used as control of experimental conditions. Each experiment was performed in triplicate, using 40 embryos per MO injected. HY: hypothalamus, TP:thyroid primordium. 


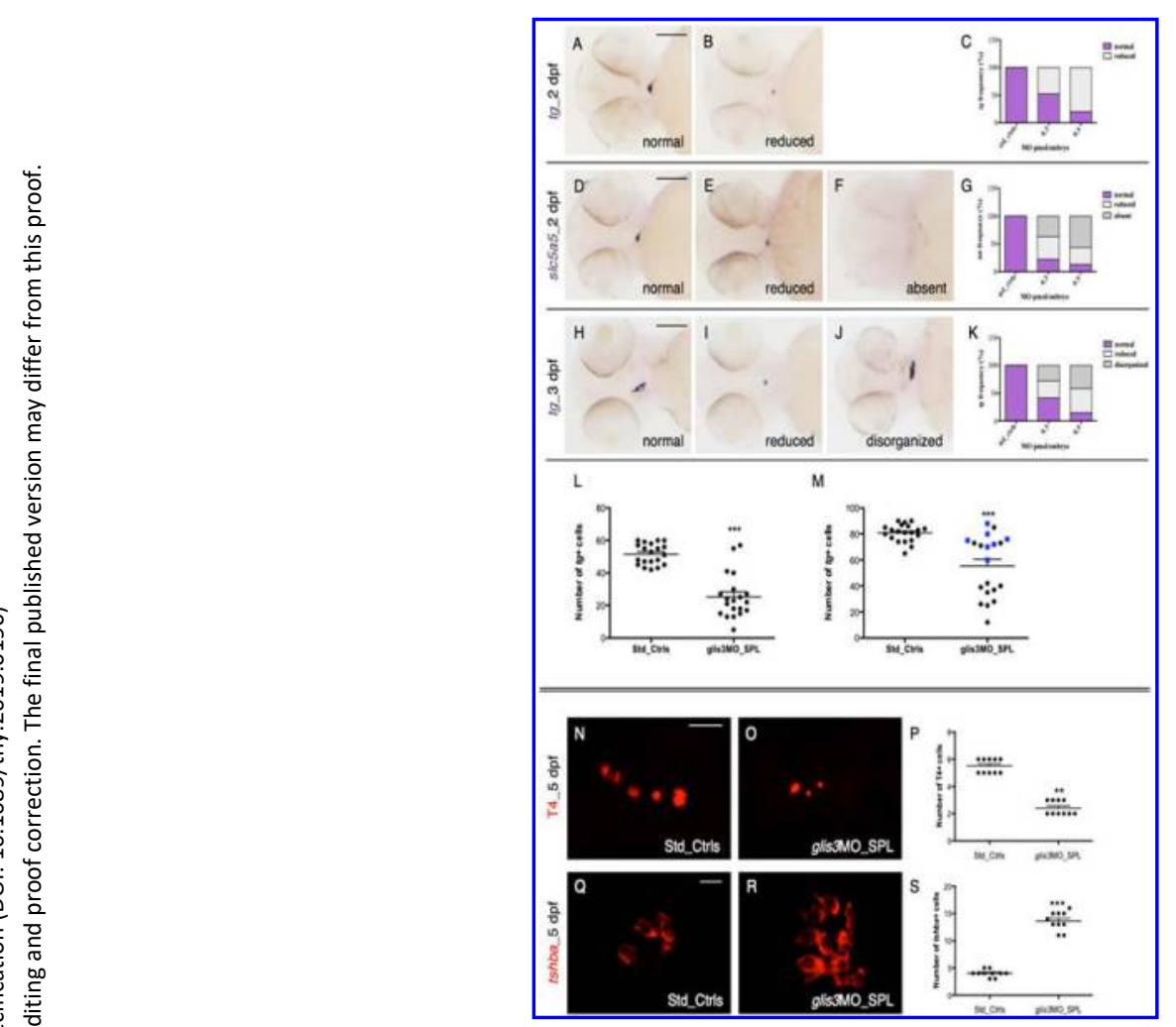

Fig 2. Analysis of development and function of thyroid gland. A and B WISH of $t g$ expression in embryos injected with Std-ctrIMO and glis3MO_SPL at 0.3 and $0.5 \mathrm{pmol} / \mathrm{e}$, at $2 \mathrm{dpf}$. D-F s/c5a5 expression in the injected embryos at $2 \mathrm{dpf}$. $\mathbf{H}-\mathbf{J}$ analysis of thyroid elongation using WISH of $t g$, in the injected embryos at $3 \mathrm{dpf}$. Graphs $\mathbf{C}, \mathbf{G}$ and $\mathbf{K}$ bars indicate the percentages of the injected embryos belonging the different phenotypic classes. Graphs $\mathbf{L}$ and $\mathbf{M}$ quantification of $t g$ cell number in control and morphant embryos at 2 and $3 \mathrm{dpf}$. Blue squares indicate morphants with a disorganized thyroid. N-O anti-T4 IHC in control and in glis3MO_SPL larvae at $0.5 \mathrm{pmol} / \mathrm{e}$, at $5 \mathrm{dpf}$. $\mathbf{P}$ count of the number of T4-producing follicles in Std_CtrIMO and in glis3MO_SPL injected larvae. Q-R WISH of tshba using FastBlue staining for the acquisition of the fluorescence signal in control and in glis3MO_SPL larvae at $0.5 \mathrm{pmol} / \mathrm{e}$, at $5 \mathrm{dpf}$. S count of the number of tshba-producing follicles in Std_CtrIMO and in glis3MO_SPL injected larvae. Each experiment was performed in triplicate, using 40 embryos per $\mathrm{MO}$ injected. For quantification ( $\mathrm{P}$ and $\mathrm{S}), 10$ embryos were used for each injection. All views were acquired in ventral view, anterior to the left. Scale bars: $250 \mu \mathrm{m}$ and100 $\mu \mathrm{m}$. Statistical significance is calculated using Student's t-test $(* * p<0.01 ; * * * p<0.001)$. 


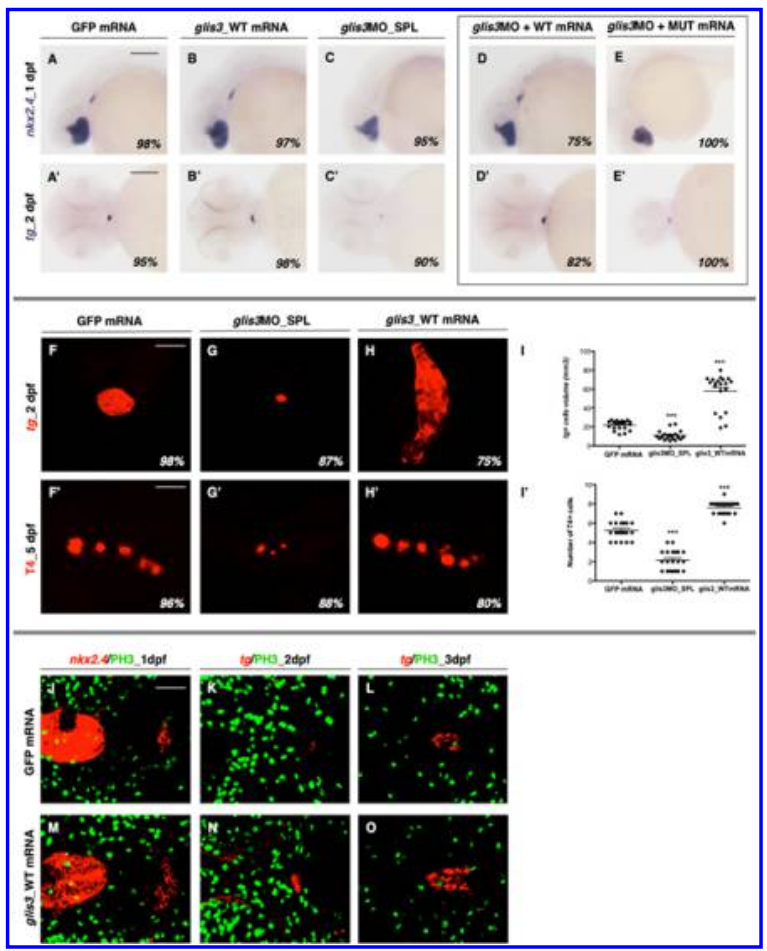

Fig 3. Rescue experiment. A-E' WISH of $n k x 2.4$ expression, at $1 \mathrm{dpf}$ and $t g$ at $2 \mathrm{dpf}$, in embryos injected with GFP mRNA (100 pg/e), glis3_WT mRNA (100 pg/e), glis3MO_SPL (0.5 pmol/e), glis3MO_SPL (0.5 pmol/e) + glis3_WT mRNA (100 pg/e), and glis3MO_SPL $(0.5$ pmol/e) + glis3_MUT mRNA (100 pg/e). Views of embryos were acquired in lateral (AE) and ventral $\left(A^{\prime}-E^{\prime}\right)$ views, anterior to the left. Scale bar: $250 \mu \mathrm{m}$. Analysis of glis3 overexpression. F-H WISH of $t g$ using FastBlue staining for the acquisition of the fluorescence signal. I Quantification of the thyroid volume $\left(\mathrm{mm}^{3}\right)$ in 20 embryos injected with GFP mRNA (250 pg/e), glis3MO_SPL (0.5 pmol/e), and glis3_WT mRNA (250 pg/e). F'$\mathbf{H}^{\prime}$ Anti-T4 IHC in the injected larvae at $5 \mathrm{dpf}$. I' count of the number of T4-producing follicles in 20 embryos injected for each condition. J-L and M-O WISH of $n k \times 2.4$ and $t g$, using FastBlue staining for the acquisition of the fluorescence signal, followed by phosphohistone H3 (PH3) IHC.

Embryos were all flat mounted and acquired in ventral view, with the anterior to the left. Scale bar: $100 \mu \mathrm{m}$. Asterisks indicate statistically significant differences (Mann-Whitney test; $* * *, p<001)$. 


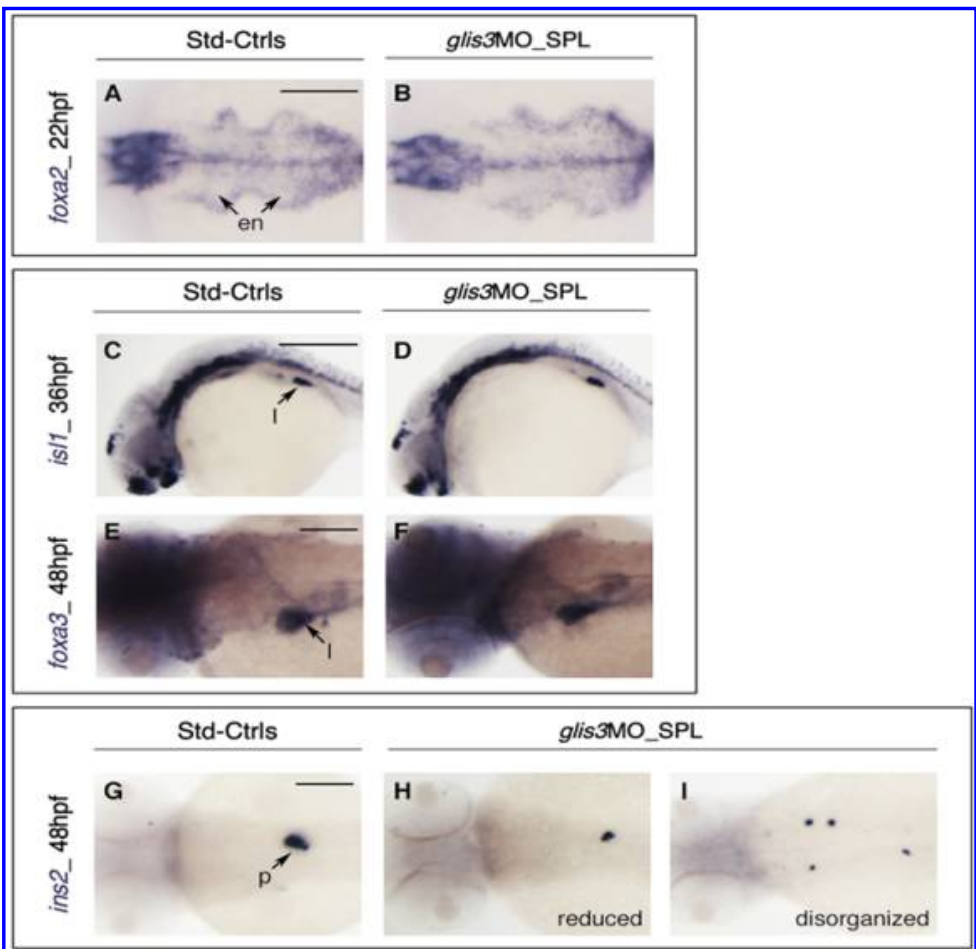

Fig 4. Analysis of pharyngeal endoderm and endoderm-derived organs in glis 3 morphants. A-B WISH showing foxa2 expression in the endoderm (en) at 22 hpf. C-F analysis of liver (I) formation by WISH of (C-D) is/1 at $36 \mathrm{hpf}$ and (E-F) foxa3 at $48 \mathrm{hpf}$. G-I analysis of pancreas (p) development by WISH of ins 2 at $48 \mathrm{hpf}$. Each experiment was performed in triplicate, using 35 embryos per MO injected. Views of embryos were acquired in dorsal (A-B, E-F and G-I) and lateral (C-D) view, anterior to the left. Scale bars: $250 \mu \mathrm{m}$ and $100 \mu \mathrm{m}$. 

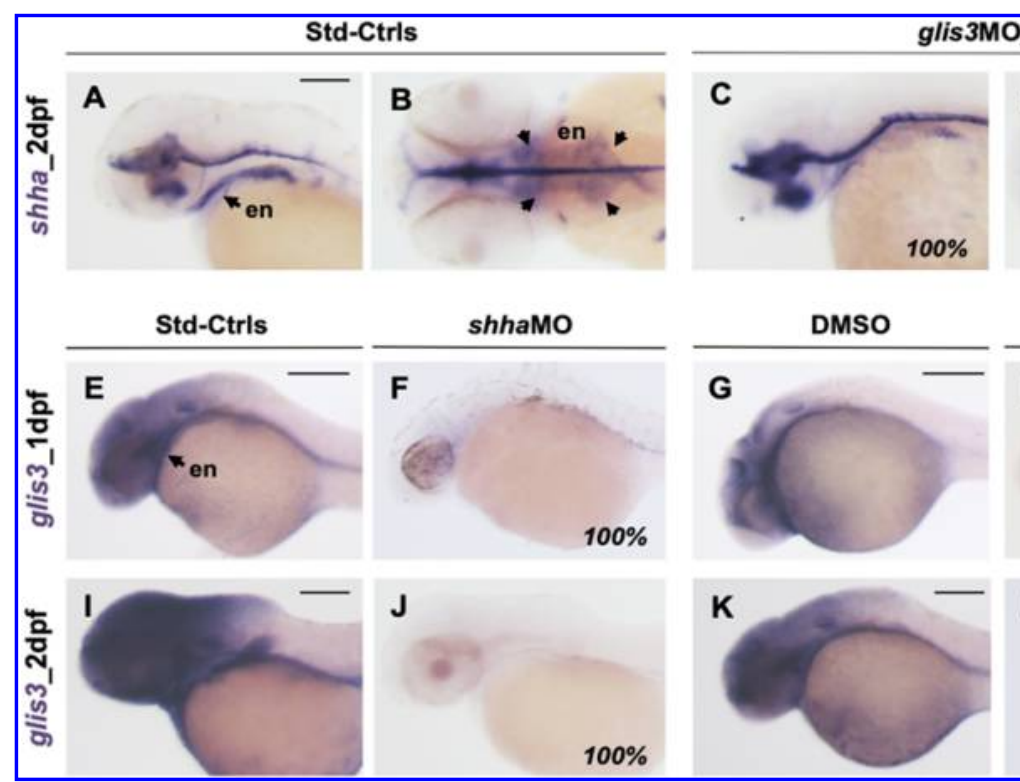

$100 \%$

D
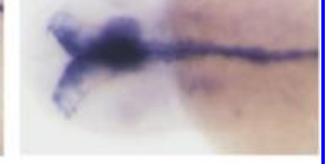

Cycl

H

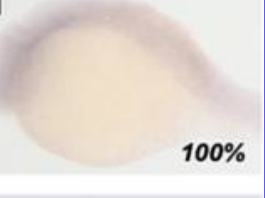

$\mathbf{L}$

$100 \%$

Fig 5. Analysis shha expression in glis3 morphants. A-D WISH of shha expression in embryos injected with Std-ctrl and glis3MO_SPL at 0.5 pmol/e. E-J analysis glis3 expression in shh deficient embryos. To blocking the activity of the Shh pathway, the shha_MO (1.25 pmol/e) and the pharmacological inhibitor cyclopamine $(10 \mu \mathrm{M})$ are used. As controls, embryos injected with Std_CtrIMO or treated with DMSO (vehicle) were used. Each experiment was performed in triplicate, using 50 embryos per each experiment. Embryos were acquired in lateral $(A, C, E-L)$ and dorsal $(B, D)$ views, anterior to the left. Scale bar: $250 \mu \mathrm{m}$. en: endoderm. 


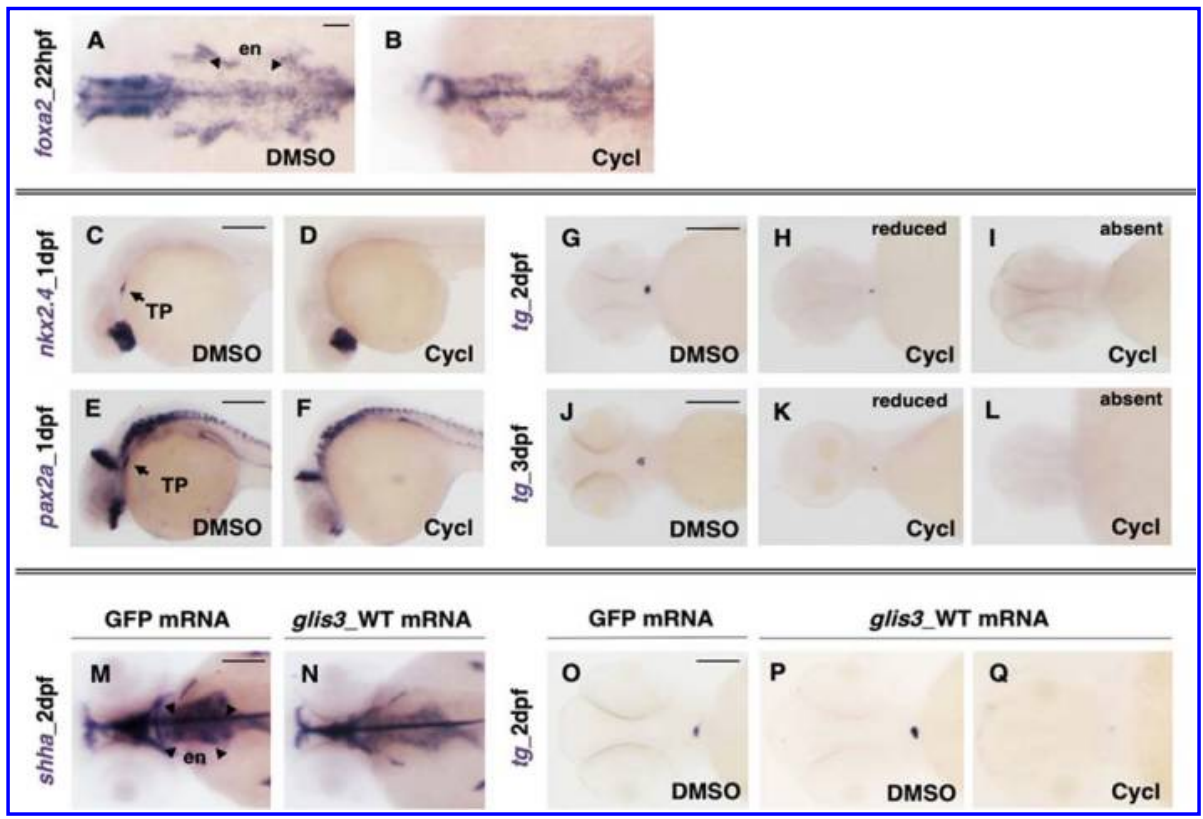

Fig 6. Analysis of thyroid gland development in cyclopamine-treated embryos. A and B WISH showing foxa2 expression in the endoderm (en) at $22 \mathrm{hpf}$ in control embryos (DMSO $1 \%)$ and embryos treated with cyclopamine $(10 \mu \mathrm{M})$. C-F WISH of $n k \times 2.4$ and pax2a expression at $1 \mathrm{dpf}$. G-L WISH of $t g$ at 2 and $3 \mathrm{dpf}$. The embryos are subdivided as normal, reduced or absent $t g$ signal. Analysis of shha in glis3 overexpressing embryos. $\mathbf{M}$ and $\mathbf{N}$ WISH of shha expression in embryos injected with GFP mRNA (250 pg) and glis3_WT mRNA (250 pg/e) at 2dpf. Cyclopamine treatment in glis3-overexpressing embryos. O-Q WISH of $t g$ in embryos injected with the GFP mRNA (250 pg) and the glis3_WT mRNA (250 $\mathrm{pg} / \mathrm{e})$, followed with treatment with DMSO or Cyclopamine $(10 \mu \mathrm{M})$. Each experiment was performed in triplicate, using 50 embryos. Views of embryos were acquired in lateral (C-F), dorsal ( $A, B, M$ and $N$ ) and ventral (G-L and $O-Q$ ) views, anterior to the left. Scale bar: 250 $\mu \mathrm{m} . T P$ : thyroid primordium, en: endoderm. 


\section{Molecular and morphological validation of glis3MO_SPL}

The glis3MO_SPL was designed to overlap with the exon2-intron2/3 boundary of the glis3 pre-mRNA, which resulted in the retention of a portion of the intron2/3. Different sets of primers were used to discriminate between the wild-type (mRNA-WT) and morphant (mRNA-MO) trancripts (Supplementary Fig 1A). The validation of morpholino performed by semiquantitave and qRT-PCR revealed that the amount of aberrant transcript (mRNA-MO) was dependent by the dose of glis3MO_SPL (0.3-1pmol/embryo) injected (Supplementary Fig $1 \mathrm{~B}$ and $\mathrm{C})$. 


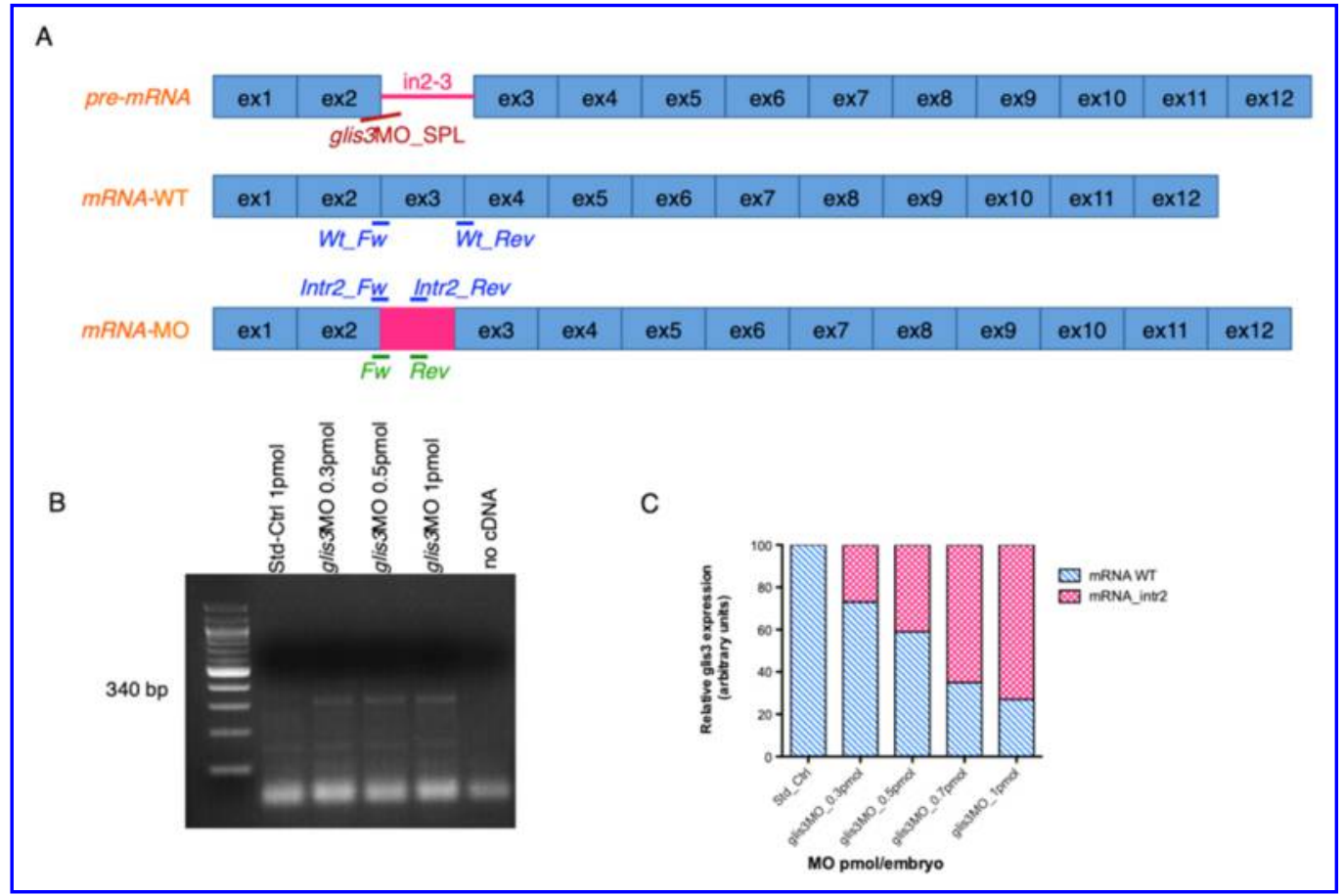

Supplementary Fig 1. Morpholino-mediated knockdown of the glis3 genes in zebrafish.sepi:A glis3 gene structure: the squared boxes correspond to the exons, the orange lines correspond to the introns. glis3SPL_MO is targeted to the splice donor site between exon 2 and intron 2-3 (E2i2-3). The position of primer sets used in RT-PCR and qRT-PCR to analyse the altered splicing are indicated in green and in blue, respectively. glis3MO_SPL was validated by RT-PCR injecting different doses $(0.3,0.5,0.7$ and 1pmol/ embryo) in the zebrafish embryos at 1-2 cells stage. The glis3SPL_MO causes the retention of a part of intron2-3 producing a PCR product of 340bp.SEPC qRT-PCR of WT (mRNA WT) and aberrant (mRNA_intr2) glis3 transcripts expressed after injec- tion of growing doses of glis3MO_SPL.

Subsequently, to chose the working doses of $\mathrm{MO}$, we analyzed different morphological parameters (embryonic length, tail curvature, cardiac edema, and head necrosis). At 1-3 dpf, the embryos were observed and classified into three different phenotypic classes (Supplementary Fig2): normal (Panels B, F, and L), similar to embryos injected with the StdCtrIMO (Panels A, E, and I); moderate (Panels C, G, and M), presenting slight alterations in body-length and tail curvature, small head and cardiac edema; severe (Panels $D, H$, and $N$ ), with gross morphological alterations, and extensive necrosis. 
Page 33 of 40

33

The 0.3 and $0.5 \mathrm{pmol} / \mathrm{embryos}$ were selected for the further experiments, because the severe class was less represented (Graph O).

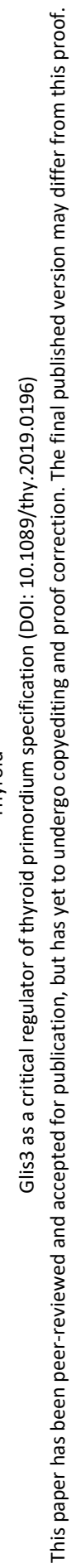


34

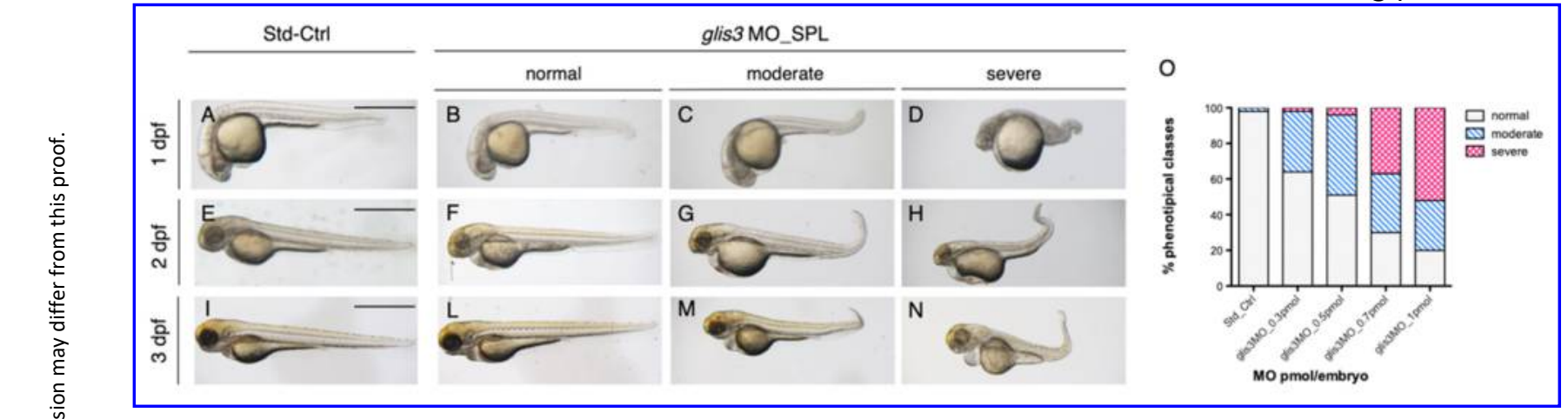

Supplementary Fig 2. Phenotypic classes of embryos injected with glis3MO_SPL.SE-PA-N

Three phenotypic classes normal, moderate and severe analysed at three different developmen- tal stages $(1,2$, and $3 \mathrm{dpf})$. The normal embryos ( $\mathrm{B}, \mathrm{F}$ and $\mathrm{L})$ are indistinguishable form the controls ( $A, E$ and I). The moderate class ( $C, G$ and $M$ ) presents embryos with curved tail, cardiac edema and impaired circulation. The severe embryos ( $D$, $\mathrm{H}$ and $\mathrm{N}$ ) are strongly affected by necrosis, car- diac edema, reduced circulation and stasis. according to the in- jected doses. Scale bar: $250 \mu \mathrm{m}$.

\section{Double fluorescent analysis of the early anche late thyroid markers}

Taking into account that the possible embryonic delay of morphants can influence the analysis, we evaluated the frequency of thyroid phenotypes at different time points. The percentage of embryos reaching the $26 \mathrm{hpf}$ or $30 \mathrm{hpf}$ with undetectable expression of $n k \times 2.4$ and pax $2 a$ passed from $75-85 \%$ to $33-10 \%$. Consequently at $30 \mathrm{hpf}$, the embryos with reduced but detectable expression of both markers represented the most frequent phenotypic class (Fig $3 \mathrm{~A}$ and B), likely due to a selection bias among the morphants. However, a reduced $t g$ signal was seen in almost $100 \%$ of morphants at later stages (Fig 2). In this class of morphants, when the double WISH was performed, pax2a signal was affected to a larger extent than $n k x 2.4$ (Fig 3D and $D^{\prime}$ ), but $75 \%$ of morphants displayed a significant reduction of both transcripts (Fig $3 E$ and $E^{\prime}$ ). In contrast with the diffuse colocalization in Std-Ctrls (Fig $3 A^{\prime \prime}$ ), only few cells co-expressed $n k \times 2.4$ and pax2a in morphants (Fig 3D" and E"). 
Between 55 and 60hpf, we observed similar thyroid phenotypic frequencies in morphants, consistent with the recovery of the developmental embryonic delay (Fig $3 \mathrm{~F}, \mathrm{G}, \mathrm{K}$ and $\mathrm{L}$ ). At $60 \mathrm{hpf}, n k \times 2.4$ and $t g$ signals were reduced to variable extent in $45 \%$ of morphants, (Fig $3 \mathrm{I}, \mathrm{I}^{\prime}, \mathrm{H} \mathrm{H}^{\prime}$ ). However, a strong reduction of both signals was seen in the remaining $55 \%$ of morphants (Fig 3J and $J^{\prime}$ ). The pax $2 a$ expression was always diminished in almost $100 \%$ of morphants (Fig $3 \mathrm{~N}$ and O) associated with a variable decrease of $\operatorname{tg}$ (Fig $3 \mathrm{~N}^{\prime}$ and $\mathrm{O}^{\prime}$ ). In glis3 morphants, the co-localization of $n k \times 2.4 / \mathrm{tg}$ or pax2a/tg staining was almost undetectable (Fig 3H"'-J" and $\mathrm{M}^{\prime \prime}-\mathrm{O}^{\prime \prime}$ ). 


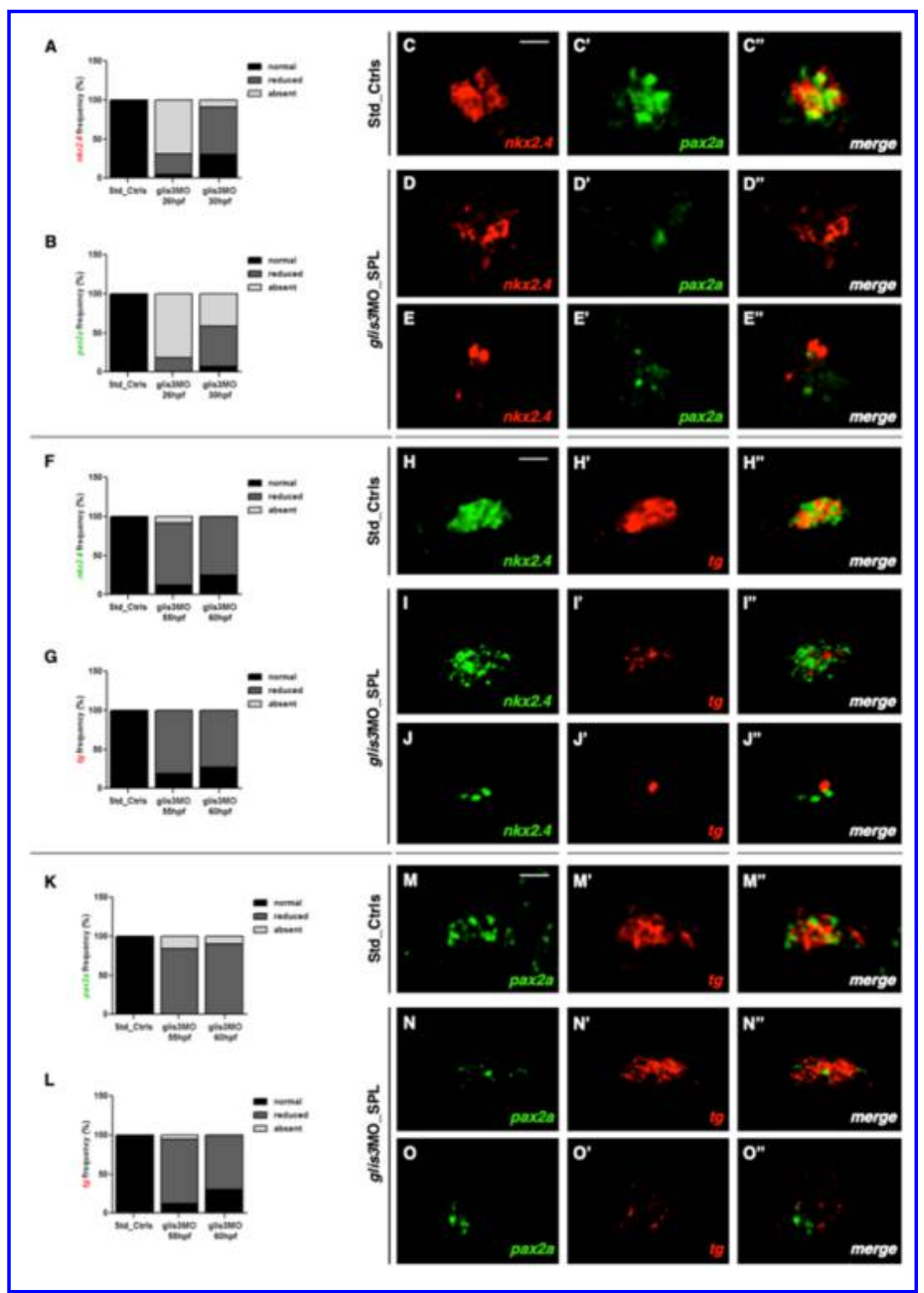

Supplementary Fig 3. Double fluorescent WISH of thyroid markers. Graphs A-B, F-G, K-L bars indicate the percentages of the injected embryos belonging the different phenotypic classes. C-E" nkx2.4/pax2a in Std_Ctrls at 26hpf and glis3 morphants at 30hpf. H-J" nkx2.4/tg in Std_Ctrls at 55hpf and glis3 morphants at 60hpf. M-O" pax2a/tg in Std_Ctrls at $55 \mathrm{hpf}$ and glis3 morphants at 60hpf. All embryos are acquired in ventral view, anterior to the top. Scale bar: $100 \mu \mathrm{m}$. The images are representative of 10 embryos per injection performed in triplicate.

\section{Molecular and morphological analysis of the alternative glis3MO2}

The second glis3MO_SPL (glis3_MO2) was designed to overlap with the intron3/exon4 boundary of the glis3 pre-mRNA, which resulted in the skipping of the exon 4. (Ref. 33, O'Hare et al., 2016). 
The morphological evaluation of the embryos injected with glis3_MO2 was performed as described before. The $0.7 \mathrm{pmol} / \mathrm{embryos}$ was selected for the further experiments, because the moderate class was well represented compared to normal and severe ones (Graph 4A).

As for the first glis3MO_SPL, we analysed the thyroid primordium development and function injected with $0.7 \mathrm{pmol} / \mathrm{e}$ of glis3_MO2 (Graph 4B-E). Notably all the obtained results well recapitulated what was obtained with the first MO. In fact, at $1 \mathrm{dpf}$ the glis3_MO2 morphants exhibited a deficient formation of thyroid primordium with a marked reduction/absent expression of both $n k \times 2.4$ and pax2a in most of the analysed embryos (4B). At $2 \mathrm{dpf}$ also the $t g$ and s/c5a5 expression was reduced or absent in the morphants (4C). Consistently, at $5 \mathrm{dpf}$, the number of T4-positive follicles was significantly reduced in morphants compared to controls (4D), associated with an increased number of the tshba-positive pituitary cells (4E). 


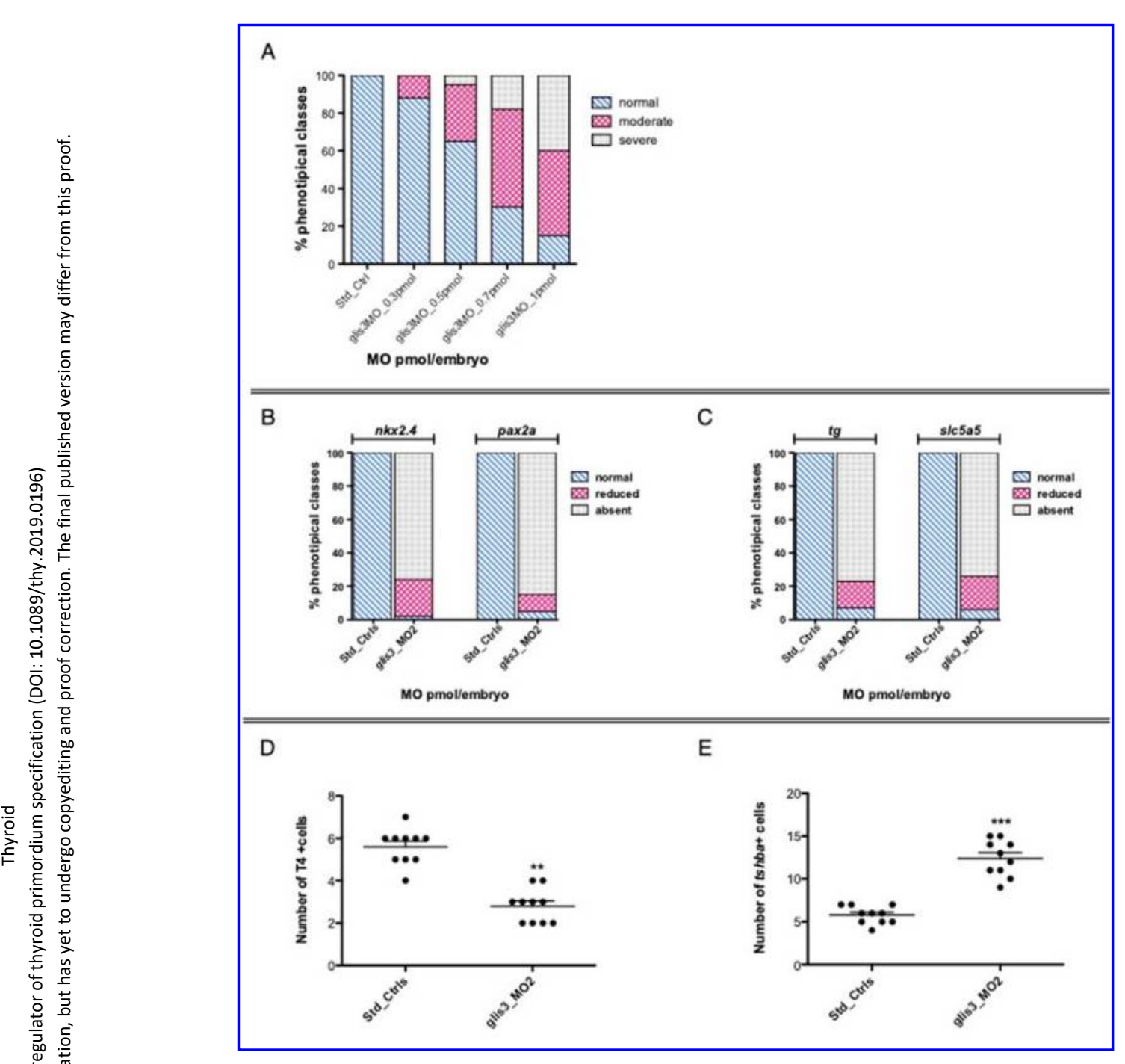

Supplementary Fig 4. Graph A Frequency of the phenotypic classes of embryos injected with glis3MO_2 Graph B and C Bars indicated the percentage of the glis3MO_2 injected embryos $(0.7 \mathrm{pmol} / \mathrm{e})$ belonging the different phenotypic classes after WISH analysis of $n k \times 2.4$, pax2a at $1 \mathrm{dpf}(\mathrm{B})$ and $\operatorname{tg}$ and s/c5a5 at $2 \mathrm{dpf}(\mathrm{C})$. Graph D and E Count of the number of T4-positive cells (D) and tshba-producing follicles (E) in Std_Ctrls and glis3_MO2 injected larvae. Statistical significance is calculated using Student's t-test $(* * p<0.01 ; * * *$ $\mathrm{p}<0.001)$.

\section{Analysis of the pancreatic acini development in the glis3 knockdown embryos}

In order to exclude a global impairment of the exocrine pancreas in the glis3 morphants, we analysed the expression of ptf1a, a specific pancreatic acini transcription factor 
(Supplementary Fig.5). The WISH analysis showed that the pancreatic acini were unaffected in the morphants (D) compared to controls (B), thus supporting the idea that glis3 is specifically involved in the endocrine pancreas development.

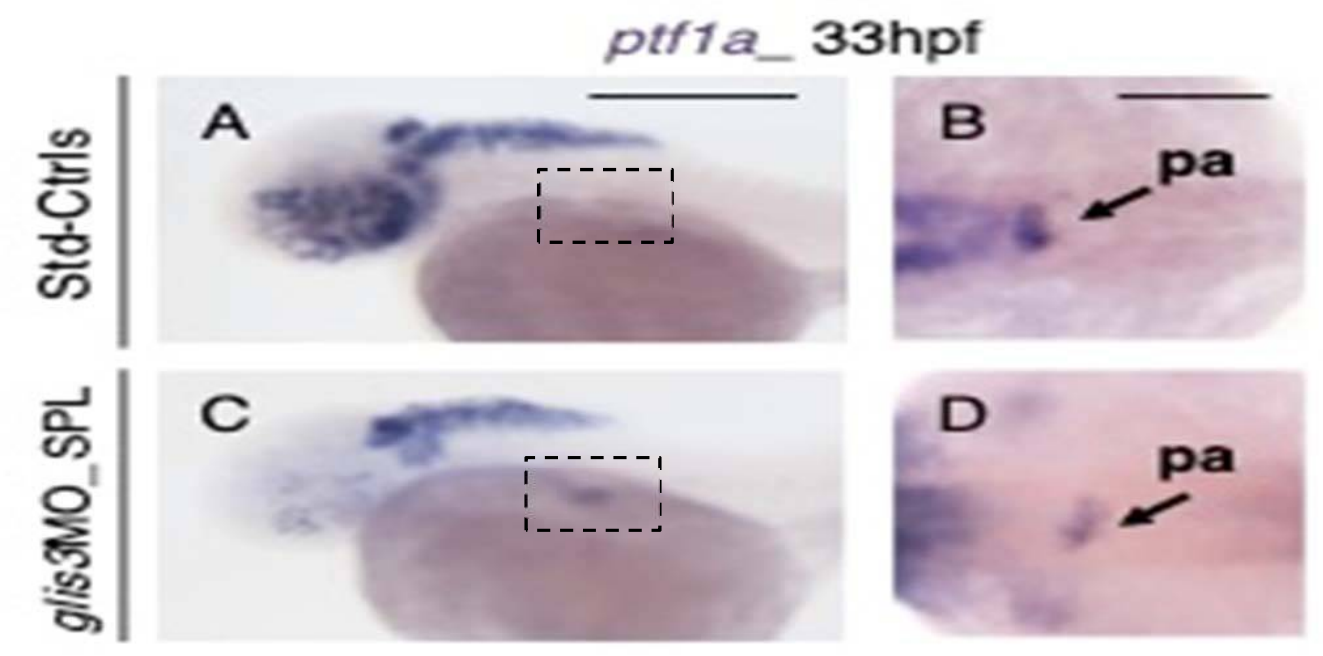

Supplementary Fig 5. Analysis of pancreatic acini development. (A-D) WISH of ptf1a expression in embryos injected with Std_CtrIMO and glis3MO_SPL at 0.5pmol/e at 33hpf. The signal was acquired in lateral $(A$ and $C$ ) and dorsal $(B$ and $D$, at higher magnification of the area of interest) view, anterior to the left. Scale bars: 250 and $100 \mu \mathrm{m}$. pa: pancreatic acini.

\section{5. glis3 and sufu zebrafish proteins interact in vitro}

To test whether and how the glis3 and sufu zebrafish proteins interact, we performed an immune-localization and co-immuno-precipitation (Co-IP). In single transfection experiments, the pcDNA4-Myc/His-glis3 localized into the nucleus (Supplementary Fig 6AC) whereas the pEGFP-sufu is visible in the cytosol (Fig 6D-F). Interestingly, both proteins co-localized into the nucleus of co-transfected HeLa cells (Fig 6G-I). The Co-IP assay confirmed that sufu and glis3 co-immunoprecipitate (Fig 6J) in a protein complex. Given the high conservation of the VYGHF motif at the N-terminus of zebrafish glis3, which is known to account for the sufu binding (44), the direct interaction of these two zebrafish proteins acquires likelihood. 


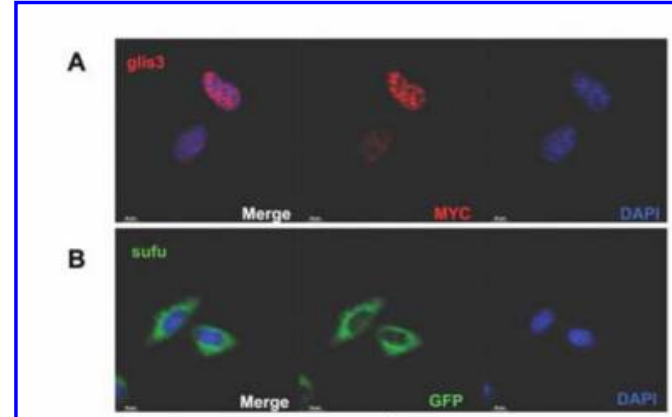

c
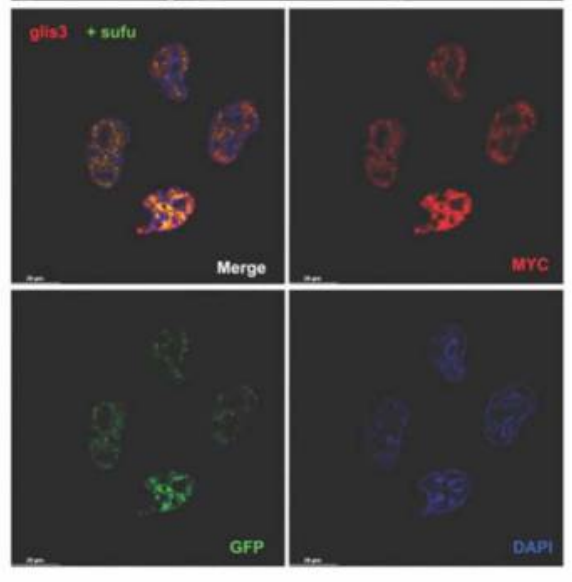

D

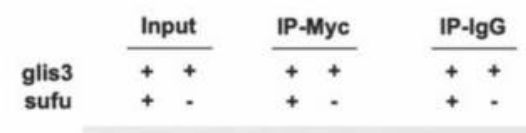

aMyc

$\longrightarrow$

$\alpha$ GFP

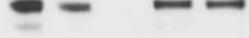
$-$

Supplementary Fig 6. Immunolocalization of the zebrafish glis3 and sufu proteins. A-C and D-F immunocytochemistry (ICC) of pcDNA4-Myc/His-glis3 and pEGFP-sufu individually transfected in HeLa cells. Glis3 colocalizes with dapi into the nucleus (C), whereas sufu presents a cytoplasmatic localization (F). G-I when cotransfected glis3 and sufu colocalize into the nucleus (I). J co-immunoprecipitation assay HeLa cells are transfected with pcDNA4-Myc/His-glis3 + pEGFP-sufu or with the glis3 plasmid alone. For each transfection the total protein content (input) and the immunoprecipitated for c-Myc or IgG (negative control) are subjected to WB analysis. The incubation with the anti-cMyc and anti-GFP antibodies is used to detect glis3 and sufu respectively. 